Research Article

\title{
Extend Disturbance Observer of Backlash-Based Robust Sliding-Mode Control
}

\author{
Weixing Wang $\mathbb{D}^{\mathrm{D}}$, Dapeng Mao, and Botong Li \\ Changchun Institute of Optics, Fine Mechanics and Physics, Academy of Sciences, Changchun 130033, China \\ Correspondence should be addressed to Weixing Wang; wangweixing@ciomp.ac.cn
}

Received 6 May 2021; Revised 30 June 2021; Accepted 19 July 2021; Published 29 July 2021

Academic Editor: Yueyang Li

Copyright ( $) 2021$ Weixing Wang et al. This is an open access article distributed under the Creative Commons Attribution License, which permits unrestricted use, distribution, and reproduction in any medium, provided the original work is properly cited.

\begin{abstract}
The backlash by the hysteresis between the input and the output is always present in the inertial stabilized platform, which will seriously affect the dynamic performance of the platform system at low speed. So, the backlash has been paid more and more attention for the use in the inertial stabilized platform. To handle such a situation, extend disturbance observer (EDOB) has a high advantage to compensate the disturbance caused by backlash. However, some research studies show that the observation effect for some fast time-varying disturbances is satisfactory, which will strict limits on the rate of change in disturbance and still hamper its application; consequently, this paper proposes a sliding-mode-based extend disturbance observer (SMEDO) to compensate backlash. By well-designed sliding-mode surface, it is unnecessary to measure the whole state and the lack of robustness against unmatched uncertainties of the resulting controller, and the robustness and accuracy of modified disturbance observer can be enhanced. Experiments were carried out on a DSP-based platform with backlash in the pitch shafting. The obtained experimental results demonstrate that the SMEDO scheme has an improved performance with the dynamic performance and shafting transmission accuracy compared with the traditional methods.
\end{abstract}

\section{Introduction}

Due to the influence of flight platform environment, the airborne inertial stabilization platform has the characteristics of small volume, light weight, and strong anti-interference performance. In the design of the drive system, one or more stages of gear transmission are usually used to realize compactness and torque drive. It needs to be a certain backlash space between the driving and driven wheels, which has the characteristics of nonlinear, nondifferentiable, and nonanalytical description. Backlash characteristics are easy to cause instability, hysteresis, even commutation jump, and impact oscillation of the platform at low speed, which is necessary to be controlled and compensated. There are several ways given to reduce the backlash effect by mechanical and control solutions. The mechanical solution consists in modifying the structure of the mechanical system, which is expensive $[1,2]$. The control solution consists in minimizing the effect of backlash by using a control law that takes into account the hysteresis disturbance [3-11]. The latter solution is less expensive.
Designing a nonlinear observer to estimate the disturber torque describing the backlash effects by using a mathematical model representing an inversed sigmoid, researcher constructs an adaptive control using a PD controller associated with the last torque observer to compensate the dead zone effects [12]. A disturbance observer (DOB) is constructed in order to estimate and compensate the disturbance in an inner feedback loop fusion, such that the outer controller is used to control the "backlash-free" system [13]. The extended state observer is improved by introducing a new nonlinear function to avoid high-frequency oscillation and simplify the designing process [14]. Although the above research has effectively reduced the influence of backlash, the dynamic model and backlash impact model of the system are highly required and the control quantity is difficult to settle. Among these problems, the nonlinear disturbance observer is used to estimate the external disturbances and model uncertainties, which is attenuation that may provide good stability and tracking performance $[15,16]$. In literature [17], a nonlinear extended disturbance observer is 
further considered to the aircraft terminal control law and missile guidance design with attack angle constraint. These studies are designed of the linear system and lack consideration for the nonlinear system or have application limitations.

Sliding-mode control (SMC) is an effective and popular control strategy for the controlling system affected by uncertainties and external unmeasurable disturbances, which has found several applications in diverse fields [18]. The most important advantage of SMC is the robustness against and insensitivity to parameter variations and external disturbances under the so-called matching conditions. A variety of SMC strategies have been proposed in the literature to address the problem of mismatched uncertainties and nonlinearity [19-22]. Combining SMC with methods of nonlinear disturbance observer is an effective means to estimate uncertainty and disturbances. This method can not only enable a reduction in the magnitude of the discontinuous component in control but also thereby reduce the switching gain so that the buffeting effect of the system can be weakened.

Therefore, in this paper, aiming at the nonlinear problem of backlash in gear transmission of inertial platform shafting, the dynamic model of the platform and the approximate model of backlash are established. On the basis of the design of the nonlinear disturbance observer and the extended disturbance observer, a robust sliding-mode principle is introduced to enhance the robustness of modified disturbance observer so that the platform changes direction at low speed and the impact of backlash transmission will be weakened. According to the idea of backlash extend disturbance observer based on a robust sliding-mode principle, system stability proof is given.

\section{The Position Servo System with Backlash}

Taking the position servo system of the inertial stabilization platform as the research object, the dynamic model of the position system is established, and the approximate dead zone model is introduced, and the pitch axis of the servo system is driven by gear. The position servo system is mainly composed of servo motor (including drive motor and reduction gear), transmission gear, encoder, load, and pitch frame. In the process of position transmission, it is considered that the backlash nonlinearity mainly occurs between the master and slave transmission gears, and the structural diagram is shown in Figure 1.

According to the analysis of the platform motion mechanism method, the dynamic equation of the position system [23] in Figure 1 is expressed as follows:

$$
\left\{\begin{array}{l}
J_{e} \ddot{\theta}_{e}+B_{e} \dot{\theta}_{e}+T_{f e}=T-\tau, \\
J_{m} \ddot{\theta}_{m}+B_{m} \dot{\theta}_{m}+T_{f m}=i \tau \\
T=K_{T} U
\end{array}\right.
$$

In equation (1), $J_{e}$ and $J_{m}$ are the motor inertia and the load inertia, $B_{e}$ and $B_{m}$ are the equivalent viscous friction coefficient of motor and load, $\theta_{e}$ is the shafting angle of

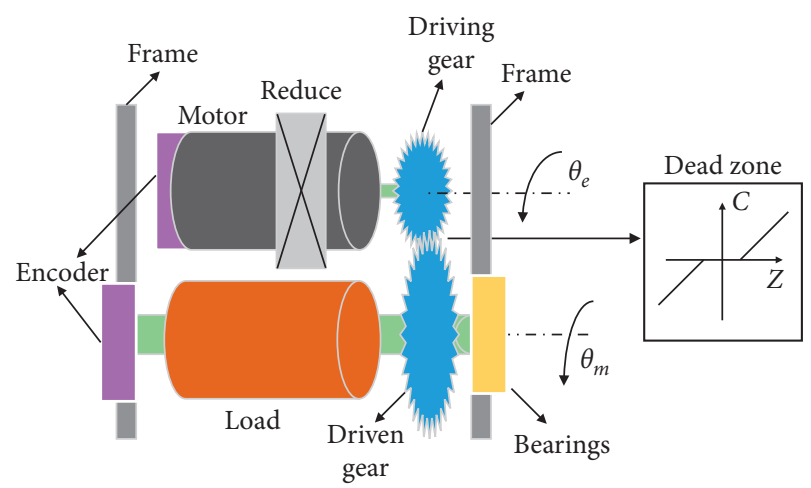

FIGURE 1: Electromechanical system including the backlash in the shafting.

motor, $\theta_{m}$ is the angle of load after gear transmission, $i$ is the transmission ratio between main and driven gears, $T_{f e}$ is the nonlinear friction of motor and modelling dynamic error, $T_{f m}$ is the nonlinear friction of load and external disturbance, $T$ is the motor output torque, $K_{T}$ is the motor torque constant, $U$ is the motor control input, and $\tau$ is the transmission torque between the master and slave gears.

The dead zone model describes the nonlinearity of the backlash by the transmission torque of the driving and driven parts of the system. The input of the dead zone model is the relative displacement between the master and slave gears, and the output is the torque. It not only reflects the torque transfer relationship between the driving and driven parts of the system but also considers the influence of system rigidity and damping. The transmission will be correct when the two bodies are in contact; in this case, the two positions are identical. When out of the contact, the transmission will be delayed with the presence of the dead zone where the relation between the body positions describes a hysteresis behaviour [24].

According to the characteristics of dead zone, the transmission torque between the master and slave gears $\tau$ can be expressed as follows:

$$
\tau= \begin{cases}K\left(z+j_{0}\right), & z<-j_{0} \\ 0, & |z| \leq j_{0} \\ K\left(z-j_{0}\right), & z>j_{0}\end{cases}
$$

In equation (2), $z=\theta_{m}-i \theta_{e}$ is the relative displacement between driving and driven gear in the backlash stage, $K$ represents the constant of rigidity, and $j_{0}$ is the dead zone amplitude. Since the dead function is not differentiable, it is not convenient to design the controller, so a continuous approximate dead function is introduced [25].

$$
\bar{\tau}=K\left(z-a j_{0} \frac{1-e^{-\gamma z}}{1+e^{-\gamma z}}\right)+d,
$$

where $\bar{\tau}$ is the approximate transmitted torque, $\gamma$ and $a$ are the constant, and $d$ is the bounded modelling error. We can decompose the expression of the approximate transmitted torque $\bar{\tau}$ to two parts as follows: 


$$
\bar{\tau}=\tau_{0}+\omega,
$$

with $\tau_{0}$ as the linear transmitted torque via a flexible link and is written as follows:

$$
\tau_{0}=K z,
$$

and $\omega$ is the disturbing and nonlinear transmitted torque as expressed by

$$
\omega=-a K j_{0} \frac{1-e^{-\gamma z}}{1+e^{-\gamma z}}+d .
$$

Since the real backlash amplitude $j_{0}$ is equal to a constant, its variation is reduced to zero. The relationship between approximate transmitted torque $\bar{\tau}$ and the relative displacement $z$ is shown in Figure 2. In the process of linearization of the backlash model, the modelling error generated exists and it is bounded.

The degree of approximate transmitted torque $\bar{\tau}$ approaching dead zone function is analysed, and the difference between them is defined as $d$, the equation of error in Figure 2 is expressed as follows:

$$
d= \begin{cases}K\left(-a j_{0}\left(\frac{1-e^{-\gamma z}}{1+e^{-\gamma z}}\right)-j_{0}\right), & z<-j_{0}, \\ K\left(z-a j_{0}\left(\frac{1-e^{-\gamma z}}{1+e^{-\gamma z}}\right)\right), & |z| \leq j_{0}, \\ K\left(-a j_{0}\left(\frac{1-e^{-\gamma z}}{1+e^{-\gamma z}}\right)+j_{0}\right), & z>j_{0} .\end{cases}
$$

When the parameter is $a=1$ and $\gamma=(2 / a)$, the area enclosed by $\bar{\tau}$ and $\tau$ is the smallest, the approximate dead zone function is the closest to the dead zone function, and the maximum value of $d$ is $d=K\left(2 j_{0} e^{-\gamma j_{0}} /\left(1+e^{-\gamma j_{0}}\right)\right)$, and there is

$$
|d| \leq K \frac{2 j_{0} e^{-\gamma j_{0}}}{1+e^{-\gamma j_{0}}}
$$
follows:

Therefore, the transmission torque can be expressed as

$$
\tau=\bar{\tau}+d=\tau_{0}+\omega+d=K\left(z-j_{0} \frac{1-e^{-2 z}}{1+e^{-2 z}}\right)+d .
$$

In the actual operation process of the platform, the equivalent viscous friction coefficient and external disturbance will change with the changes of temperature, lubrication conditions, parts wear, and load state. Therefore, when the system parameters change, equation (1) can be changed to

$$
\left\{\begin{array}{l}
J_{e} \ddot{\theta}_{e}+\left(\widehat{B}_{e}+\Delta B_{e}\right) \dot{\theta}_{e}+T_{f e}=K_{T} U-\tau, \\
J_{m} \ddot{\theta}_{m}+\left(\widehat{B}_{m}+\Delta B_{m}\right) \dot{\theta}_{m}+T_{f m}=i \tau .
\end{array}\right.
$$

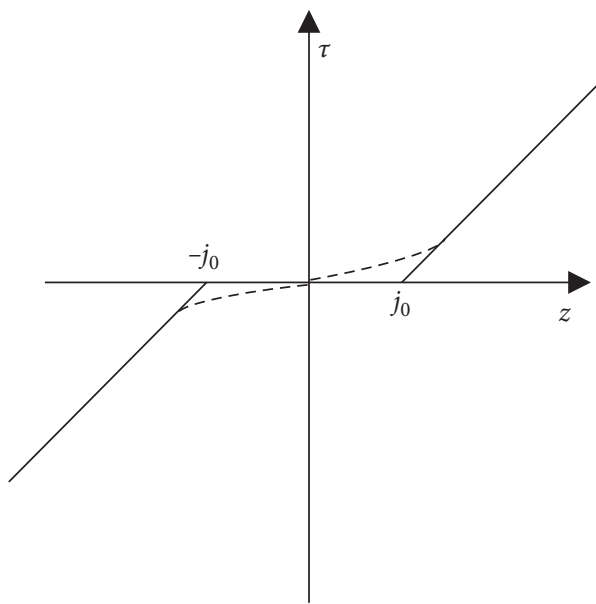

FIGURE 2: Noncontinuous backlash model.

In equation (10), $\widehat{B}_{e}$ and $\widehat{B}_{m}$ are the nominal part of $B_{e}$ and $B_{m}, \Delta B_{e}$ and $\Delta B_{m}$ are the variable part of $B_{e}$ and $B_{m}$, and the sum of unknown disturbances is defined as follows:

$$
\left\{\begin{array}{l}
T_{d 1}=\Delta B_{e} \dot{\theta}_{e}+T_{f e}, \\
T_{d 2}=\Delta B_{m} \dot{\theta}_{m}+T_{f m} .
\end{array}\right.
$$

Equivalent disturbances $T_{d 1}$ and $T_{d 2}$ mainly include system parameter variation, nonlinear friction, and external disturbance. Equation (10) can be rewritten as follows:

$$
\left\{\begin{array}{l}
J_{e} \ddot{\theta}_{e}+\widehat{B}_{e} \dot{\theta}_{e}+T_{d 1}=K_{T} U-\tau, \\
J_{m} \ddot{\theta}_{m}+\widehat{B}_{m} \dot{\theta}_{m}+T_{d 2}=i \tau .
\end{array}\right.
$$

The overall block diagram of the position servo system of inertial stabilization platform control is shown in Figure 3. System external interference $d$ is $T_{d 1}+T_{d 2}$ in the figure.

According to equations (12) and (9), the approximate expression of gear transmission torque is substituted into the motor transmission model, and the backlash dead zone system model can be obtained as follows:

$$
\left\{\begin{array}{l}
J_{e} \ddot{\theta}_{e}+\widehat{B}_{e} \dot{\theta}_{e}+T_{d 1}=U-\bar{\tau}, \\
J_{m} \ddot{\theta}_{m}+\widehat{B}_{m} \dot{\theta}_{m}+T_{d 2}=i(\bar{\tau}+d) .
\end{array}\right.
$$

In equation (13), the transmission torque of the driving gear is obtained as (14). The approximate dead zone model is adopted, and there is an approximate model error in the dynamic model of the driven gear.

$$
\bar{\tau}=U-J_{e} \ddot{\theta}_{e}-\widehat{B}_{e} \dot{\theta}_{e}-T_{d 1} .
$$

Equation (14) is substituted into the driven gear dynamic model, and we can get

$$
J_{m} \ddot{\theta}_{m}+\widehat{B}_{m} \dot{\theta}_{m}=i U-i J_{e} \ddot{\theta}_{e}-i \widehat{B}_{e} \dot{\theta}_{e}-i T_{d 1}+i d-T_{d 2} \text {. }
$$

Set $x=\dot{\theta}_{m}$, and rewrite equation (15). 


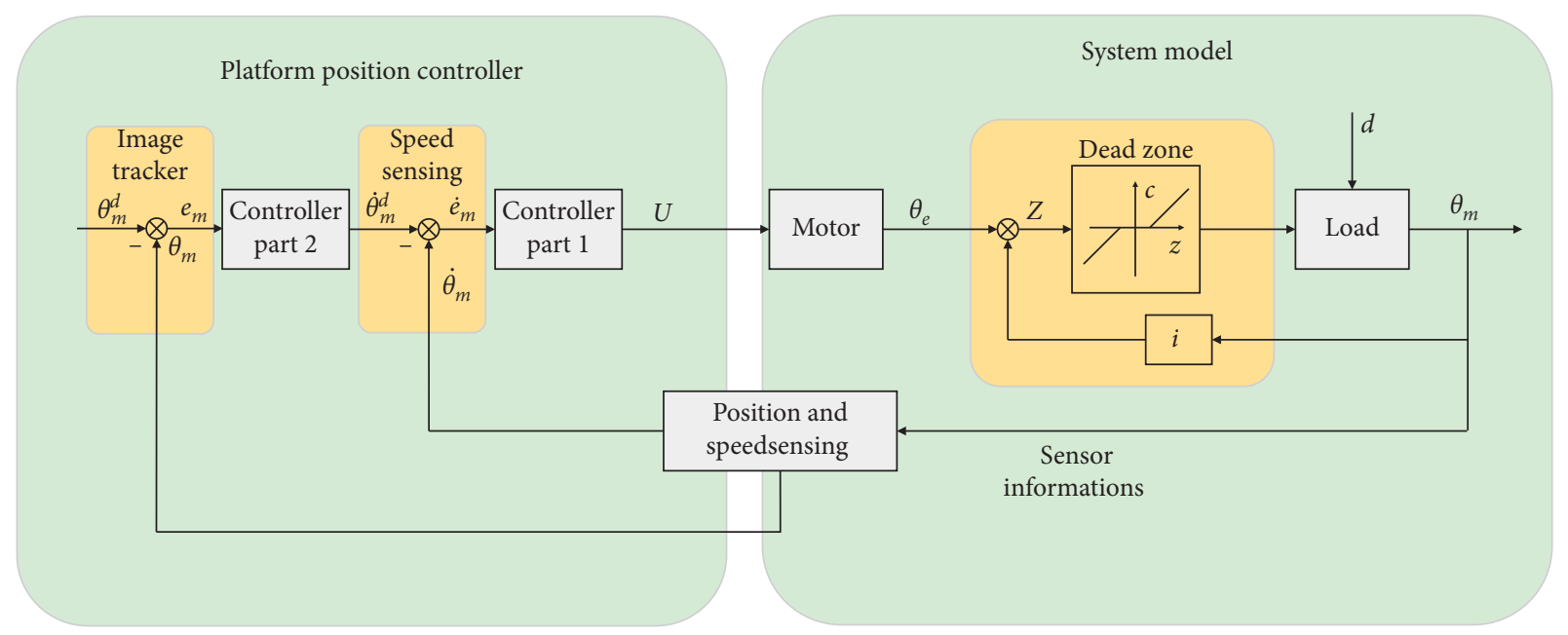

FIGURE 3: Block diagram of the position servo system of inertial stabilization platform control.

$$
\dot{x}=-\frac{\widehat{B}_{m}}{J_{m}} x+\frac{i}{J_{m}} U-\frac{i J_{e} \ddot{\theta}_{e}}{J_{m}}-\frac{i \widehat{B}_{e}}{J_{m}} \dot{\theta}_{e}+\frac{-i T_{d 1}+i d-T_{d 2}}{J_{m}} .
$$

According to equation (16), through the motor encoder combined with the motor speed reduction ratio, the integration can obtain $\ddot{\theta}_{e}, \dot{\theta}_{e}$, and $\theta_{e}$, and $\widehat{B}_{m}, \widehat{B}_{e}$, and $J_{m}$ are the known quantity, and rewrite equation (15) as follows:

$$
\dot{x}=A x+B+C U+\tilde{d}
$$

where

$$
\begin{aligned}
& A=-\frac{\widehat{B}_{m}}{J_{m}}, \\
& B=-\frac{i J_{e} \ddot{\theta}_{e}-\frac{i \widehat{B}_{e}}{J_{m}} \dot{\theta}_{e},}{C=\frac{i}{J_{m}},} \\
& \tilde{d}=\frac{-i T_{d 1}+i d-T_{d 2}}{J_{m}} .
\end{aligned}
$$

\section{Design of Extend Disturbance Observer (EDOB)-Based Robust Sliding-Mode Control}

Model mismatch, dead zone, and external disturbances widely existed in control practice of nonlinear systems. To handle such a situation, extend disturbance observer (EDOB)-based robust sliding-mode control and rapid nonlinear tracking differentiator is proposed in this paper. The brief idea is to combine a traditional nonlinear extended disturbance observer to compensate backlash dead zone nonlinearity with the principle of sliding mode. By welldesigned sliding-mode surface, the robustness of modified disturbance observer can be enhanced and the position transmission accuracy of the servo system is improved. SMC is essentially a kind of nonlinear control method, and its nonlinearity is expressed as the discontinuity of the control variables. There are some disadvantages which are the necessity to measure the whole state and the lack of robustness against unmatched uncertainties of the resulting controller.

3.1. Nonlinear Disturbance Observer. A typical nonlinear disturbance observer is designed as follows [18]:

$$
J(\theta) \ddot{\theta}+G(\theta, \dot{\theta})=U+d(t, x, u),
$$

where $U$ is the control and $d(t, x, u)$ represents the system uncertainty, parameter disturbance, and external disturbance. Suppose $d(t)$ is bounded and there is a constant $\mu>0$, letting

$$
\left|\frac{d^{i} \mathrm{~d}(t)}{\mathrm{d} t^{i}}\right| \leq \mu, \quad i=0,1,2, \ldots, r+1
$$

Remark 1 (see $[17,18]$ ). In the second-order system, the disturbance in the system is bounded and defined by $d^{*}=\sup _{t>0}|d(t)|$, and the derivative of the disturbance in the system is bounded and satisfies $\lim _{t \rightarrow \infty} \dot{d}(t)=0$, so it is proposed to consider an $n$th order single input system, and the disturbance $d(t)$ is continuous and satisfies $\left|d^{i} \mathrm{~d}(t) / \mathrm{d} t^{i}\right| \leq \mu$, that it is not required to know the bound $\mu$.

Because $\theta$ can be measured directly by no sensors, it is very difficult to get $\ddot{\theta}$ through the differential method. Thus, an auxiliary variable $\chi=\widehat{d}-p(\theta, \dot{\theta})$ is defined, Then, we get a nonlinear disturbance observer as follows [22]:

$$
\left\{\begin{array}{l}
\chi=\widehat{d}-p(\theta, \dot{\theta}), \\
\dot{\chi}=L(\theta, \dot{\theta})(G(\theta, \dot{\theta})-U)-L(\theta, \dot{\theta})(\chi+p(\theta, \dot{\theta})) .
\end{array}\right.
$$

Block diagram of the nonlinear disturbance observer is shown in Figure 4, $L(\cdot)$ is the estimator gain, and disturbance $d$ is $\widetilde{d}=\left(\left(-i T_{d 1}+i d-T_{d 2}\right) / J_{m}\right)$ in the position servo system. Combined with system dynamic equation 


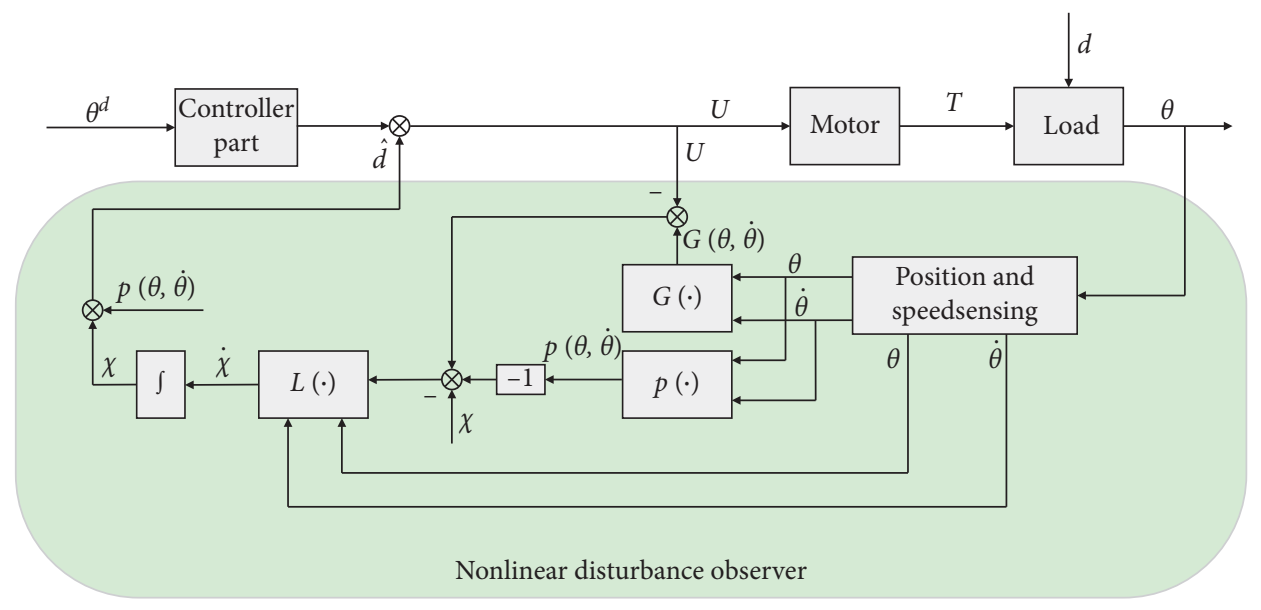

FIGURE 4: Block diagram of the nonlinear disturbance observer.

$\dot{x}=A x+B+C U+\tilde{d}$, further a nonlinear extended disturbance observer can be obtained.

$$
\left\{\begin{array}{l}
\widehat{\widetilde{d}}=z_{1}+p_{1}(x), \\
\dot{z}_{1}=-L_{1}(x)(A x+B+C U+\widehat{\widetilde{d}})+\dot{\widetilde{\tilde{d}}} \\
\vdots \\
\hat{\widetilde{d}}^{(r)}=z_{r+1}+p_{r+1}(x), \\
\dot{z}_{r+1}=-L_{r+1}(x)(A x+B+C U+\widehat{\widetilde{d}}),
\end{array}\right.
$$

where $L_{1}(x)=\left(\partial p_{1}(x) / \partial x\right), \ldots, L_{r+1}(x)=\left(\partial p_{r+1}(x) / \partial x\right)$.

Based on the design method of nonlinear expansion disturbance observer and the principle of sliding mode, considering the following uncertain nonlinear system equations, the nonlinear disturbance observer is improved:

$$
\dot{\tilde{d}}=L(\cdot)(A x+B+C U-\widehat{\widetilde{d}})+v,
$$

where $v$ is sliding-mode control amount expressed as follows:

$$
\left\{\begin{array}{l}
v=K \operatorname{sat}(s), \\
s=h(e), \\
e=x-\omega, \\
\dot{\omega}=A x+B+C U+\widehat{\tilde{d}},
\end{array}\right.
$$

where $v=K \operatorname{sat}(s)= \begin{cases}s, & |s| \leq \varphi \\ 1, & |s|>\varphi\end{cases}$

3.2. Robust Sliding-Mode Controls. SMC is a switching characteristic of the system "structure" changing with time, which can force the system to move up and down with small amplitude and high frequency along the specified state trajectory under certain characteristics. This kind of sliding mode can be designed and independent of the system parameter and disturbances. For the inertia of the system, the time lag of the switch and the error of the state detection causes the chattering phenomenon of the sliding-mode variable structure control. The occurrence of the chattering phenomenon will affect the accuracy of the system and may also stimulate the strong vibration of the unmodeled part of the system, which will cause damage to the servo system. To solve this problem, the buffeting problem can be reduced by adjusting the nonlinear observation value into the parameter value in the sliding mode [26].

In the state equation (17) of the servo system combined with the backlash dead zone model, the improved nonlinear disturbance observer to compensate backlash dead zone nonlinearity with the principle of sliding mode is expressed as follows:

$$
\left\{\begin{array}{l}
v=k e+h \dot{e}+\eta \operatorname{sat}(s), \\
s=c e+\kappa \widehat{\tilde{\tilde{d}}}, e=x-\omega, \dot{\omega}=A x+B+C U+\widehat{\widetilde{d}} .
\end{array}\right.
$$

In equation (25), $x=\dot{\theta}_{m}$ is the output shaft angular velocity of the gear driving position servo system and error is $e=\dot{\theta}_{m}-\omega . k, h$, and $\kappa$ are the control parameters to be designed. $\eta$ is the switching gain to be designed.

In order to prove the convergence stability of siding mode, some assumptions are made [17]:

(i) There is only one gear transmission device in the position servo system, and the gear reducer in the servo motor assumes that there is no backlash in the design or can be ignored compared with the shafting transmission.

(ii) The driving shaft encoder and driven shaft encoder have high accuracy, and the error caused by measuring angle can be ignored.

(iii) The disturbing term in the system is $\tilde{d}=\left(\left(-i T_{d 1}+\right.\right.$ $\left.\left.i d-T_{d 2}\right) / J_{m}\right)$ such as backlash error, system modelling error, and friction error. It is assumed that $\widetilde{d}$ is bounded and satisfies $\widetilde{d}=\sup _{t>0}|d(t)|$.

(iv) The derivative of the disturbance in the system is bounded and satisfies $\lim _{t \rightarrow \infty} \tilde{\vec{d}}(t)=0$.

(v) Choosing the appropriate observer gain makes $l>0$, $\dot{e}(t)+l e(t)=0$, which is globally stable. 
(vi) The error of interference estimation is bounded, $e^{*}=\sup _{t>0}|e(t)|$.

In equation (25), the control value of sliding mode in the NDOB was expressed as follows:

$$
v=k e+h \dot{e}+\eta \operatorname{sat}(s),
$$

and sliding-mode function is $s=c e+\tau \widehat{\widetilde{d}}$ where $\tau>0$, and we can get the derivative of $s$ as follows:

$$
\dot{s}=c \dot{e}+\tau \dot{\widetilde{\tilde{d}}}=c(\dot{x}-\dot{\omega})+\tau \dot{\widetilde{\tilde{d}}}
$$

According to the assumption that $\dot{e}(t)=-l e(t)$ is known, the disturbance observation error is set to $e_{d}=\widetilde{d}-\widehat{\widetilde{d}}$, where $\widetilde{d}$ is the true value of the disturbance observer and $\widetilde{\tilde{d}}$ is the estimated value, and $\dot{\overrightarrow{\tilde{d}}}=l e_{d}$ can be obtained with the assumption $\lim _{t \rightarrow \infty} \dot{\tilde{d}}(t)=0$ [18].

$$
\begin{aligned}
\dot{s}= & c \dot{e}+\tau \dot{\overrightarrow{\tilde{d}}}=c((A x+B+C U+\tilde{d}) \\
& -(A x+B+C u+\widehat{\widetilde{d}}))+\tau \dot{\overrightarrow{\tilde{d}}}, \\
\dot{s}= & c(\tilde{\bar{d}}-\hat{\widetilde{d}})+\tau \hat{\tilde{d}}
\end{aligned}
$$

As $\dot{\overrightarrow{\tilde{d}}}=l e_{d}^{\sim}$, further we can get

$$
\dot{s}=c(\tilde{d}-\widehat{\widetilde{d}})+\tau l e_{d}=(c+\tau l) e_{d} .
$$

At last, Lyapunov function is selected as follows:

$$
V=\frac{1}{2} s^{2}+\frac{1}{2} \Delta d^{2}
$$

where $\Delta d=\tilde{d}-\widehat{\widetilde{d}}$, and

$$
V=V_{1}+V_{2}=\frac{1}{2} s^{2}+\frac{1}{2} \Delta d^{2}
$$

In the above, there are two parts of Lyapunov function: $V_{1}$ and $V_{2}$.

Then,

$$
\dot{V}_{1}=s \dot{s}=(c+\tau l) e_{d} s \leq(c+\tau l) e_{d}^{*}|s|=\sqrt{2}(c+\tau l) e_{d}^{*} \sqrt{V} .
$$

Therefore, if $c+\tau l<0$, as $e_{d}^{*}$ is position definite, then $\dot{V}_{1}$ is negative definite.

Then, $\Delta \dot{d}=-l \Delta d$ is taken into $\dot{V}_{2}$.

$$
\dot{V}_{2}=\Delta \dot{d} \Delta d=-l \Delta d^{2} .
$$

Therefore, if $l>0$, as $l \Delta d^{2}$ is position definite, then $\dot{V}_{2}$ is negative definite.

To sum up, when $c+\tau l<0$ and $l>0$, derivatives of $V_{1}$ and $V_{2}$ are negative, so the sum of them is $V=(1 / 2) s^{2}+$ $(1 / 2) \Delta d^{2}>0$, and the derivative expression is as follows:

$$
\dot{V}=\dot{V}_{1}+\dot{V}_{2}=\sqrt{2}(c+\tau l) e_{d}^{*} \sqrt{V}-l \Delta d^{2}<0 .
$$

According to the related literature of the extended observer, the improved nonlinear disturbance observer can effectively estimate the disturbance with the unknown upper bound of the disturbance rate, but it cannot estimate the rate of its change accurately. For the position servo system with backlash, approximate transmitted torque $\bar{\tau}$ is the main factor of disturbance, estimating the rate of its change has the advantage of avoiding collision and making the position angle transmission more stable. A nonlinear extended disturbance observer was designed on the principle of highorder Taylor polynomial approximation reconstruction disturbance [27].

3.3. The Designed Extend Disturbance Observer. The designed disturbance observer is as follows [22]:

$$
\left\{\begin{array}{l}
\dot{\omega}=A x+B+C U+\widehat{\widetilde{d}}, \\
\widehat{\widetilde{d}}=\omega_{1}+p_{1}(x), \\
\dot{\omega}_{1}=-l_{1}(x) \dot{\omega}+v_{1}+\dot{\overrightarrow{\tilde{d}}} \\
\vdots \\
\hat{\widetilde{d}}^{(r)}=\omega_{r+1}+p_{r+1}(x), \dot{\omega}_{r+1}=-l_{r+1}(x) \dot{\omega}+v_{r+1},
\end{array}\right.
$$

where $p_{1}(x), \ldots, p_{r+1}(x)$ is the function vector to be designed and $l_{1}(x), \ldots, l_{r+1}(x)$ is the adjustable parameter used a bandwidth rate or bandwidth of the observer.

Set

$$
\begin{aligned}
& \Delta=\left[\begin{array}{c}
v \\
\Delta_{0} \\
\Delta_{1} \\
\vdots \\
\Delta_{r}
\end{array}\right]=\left[\begin{array}{c}
x-\omega \\
\tilde{d}-\hat{\widetilde{d}} \\
\dot{\tilde{d}}-\dot{\overrightarrow{\tilde{d}}} \\
\vdots \\
\tilde{d}^{(r)}-\widehat{\tilde{d}}^{(r)}
\end{array}\right], \\
& \dot{\Delta}=\left[\begin{array}{c}
\dot{v} \\
\dot{\Delta}_{0} \\
\dot{\Delta}_{1} \\
\vdots \\
\dot{\Delta}_{r}
\end{array}\right]=\left[\begin{array}{c}
\dot{x}-\dot{\omega} \\
\dot{\vec{d}}-\dot{\overrightarrow{\tilde{d}}} \\
\ddot{\widetilde{d}}-\ddot{\widetilde{\vec{d}}} \\
\vdots \\
\tilde{d}^{(r+1)}-\hat{\widetilde{d}}^{(r+1)}
\end{array}\right]
\end{aligned}
$$

where $\dot{\Delta}_{t}= \begin{cases}\Delta_{t}-L_{t}(x) \Delta_{0}-v_{t}, & t<r, \\ \widetilde{d}^{(t+1)}-L_{t}(x) \Delta_{0}-v_{t}, & t=r .\end{cases}$

Then, we can get following equation: 


$$
\dot{\Delta}=\left[\begin{array}{c}
\dot{v} \\
\dot{\Delta}_{0} \\
\dot{\Delta}_{1} \\
\vdots \\
\dot{\Delta}_{r}
\end{array}\right]=\left[\begin{array}{c}
\dot{x}-\dot{\omega} \\
\dot{\tilde{d}}-\dot{\overrightarrow{\tilde{d}}} \\
\ddot{\tilde{d}}-\ddot{\overrightarrow{\tilde{d}}} \\
\vdots \\
\tilde{d}^{(r+1)}-\widehat{\tilde{d}}^{(r+1)}
\end{array}\right]=\left[\begin{array}{c}
\Delta_{0} \\
\Delta_{1}-L_{1}(x) \Delta_{0}-k_{1} e-h_{1} \dot{e}-\eta_{1} \operatorname{sat}(s) \\
\Delta_{2}-L_{2}(x) \Delta_{0}-k_{2} e-h_{2} \dot{e}-\eta_{2} \operatorname{sat}(s) \\
\vdots \\
\tilde{d}^{(r+1)}-L_{r}(x) \Delta_{0}-k_{r} e-h_{r} \dot{e}-\eta_{r} \operatorname{sat}(s)
\end{array}\right] .
$$

So, we can get

$$
\text { where }
$$

$$
\dot{\Delta}=M \Delta+N \widetilde{d}^{(r+1)}+K \widetilde{e}+H \widetilde{\dot{e}}+Y S,
$$

$$
\begin{aligned}
& M=\left[\begin{array}{ccccc}
0 & 1 & 0 & \cdots & 0 \\
0 & -L_{1}(x) & 1 & \ddots & \vdots \\
0 & -L_{2}(x) & 0 & \ddots & 0 \\
\vdots & \vdots & \vdots & \ddots & 1 \\
0 & -L_{r}(x) & 0 & \cdots & 0
\end{array}\right], \\
& N=\left[\begin{array}{lllll}
0 & 0 & \cdots & 0 & 1
\end{array}\right]^{T} \text {, } \\
& K \widetilde{e}=\left[\begin{array}{lllll}
0 & & & & \\
& k_{1} & & & \\
& & k_{2} & & \\
& & & \ddots & \\
& & & & k_{r}
\end{array}\right]\left[\begin{array}{c}
0 \\
e \\
e \\
\vdots \\
\end{array}\right. \\
& H \widetilde{\dot{e}}=\left[\begin{array}{lllll}
0 & & & & \\
& h_{1} & & & \\
& & h_{2} & & \\
& & & \ddots & \\
& & & & h_{r}
\end{array}\right]\left[\begin{array}{c}
0 \\
\dot{e} \\
\dot{e} \\
\vdots \\
\dot{e}
\end{array}\right] \text {, } \\
& Y S=\left[\begin{array}{lllll}
0 & & & & \\
& \eta_{1} & & & \\
& & \eta_{2} & & \\
& & & \ddots & \\
& & & & \eta_{r}
\end{array}\right]\left[\begin{array}{c}
0 \\
\operatorname{sat}(s) \\
\operatorname{sat}(s) \\
\vdots \\
\operatorname{sat}(s)
\end{array}\right] .
\end{aligned}
$$

For any given symmetric positive definite matrix $Q$, a symmetric positive definite matrix $P$ can always be found to make $M^{T} P+P M=-Q_{0}$. Taking the Lyapunov function $V=\Delta^{T} P \Delta$, its derivative is as follows:

$$
\begin{aligned}
\dot{V} & =\dot{\Delta}^{T} P \Delta+\Delta^{T} P \dot{\Delta} \\
& =\left(\Delta^{T} M^{T}+\left(\tilde{d}^{(r+1)}\right)^{T} N^{T}+\widetilde{e}^{T} K^{T}+\widetilde{e}^{T} H^{T}+S^{T} Y^{T}\right) P \Delta+\Delta^{T} P\left(M \Delta+N \widetilde{d}^{(r+1)}+K \widetilde{e}+H \tilde{e}+Y S\right) \\
& =-\Delta^{T} Q \Delta+2 \Delta^{T} P N \tilde{d}^{(r+1)}+2 \Delta^{T} P K \widetilde{e}+2 \Delta^{T} P H \tilde{e}+2 \Delta^{T} P Y S .
\end{aligned}
$$


According to the stability analysis of sliding-mode value, it can be seen that $e$ is a bounded, there is $\|e\| \leq \alpha$, $\|\operatorname{sat}(s)\| \leq \beta$, the derivative of $e$ in the system is bounded and satisfies $\lim _{t \rightarrow \infty} \dot{e}(t)=0$, and $\left|d^{i} \mathrm{~d}(t) / \mathrm{d} t^{i}\right| \leq \mu$ is known above.

When $e \neq 0$,

$$
\dot{V} \leq-\lambda_{m}\|\Delta\|^{2}+2\|\Delta\|(\|P N\| \mu+\|P K\| \alpha+\|P Y\| \beta) .
$$

The $\lambda_{m}$ is the minimum eigenvalue of $Q, K$, and $Y$, and it is designed to make $(\|P N\| \mu+\|P K\| \alpha+\|P Y\| \beta)<0$, the norm of the extend nonlinear disturbance observer with slidingmode error will gradually converge to zero, and the extend nonlinear disturbance observer based on the sliding mode is shown in Figure 5.

\section{Implementation of Experimental System}

The backlash position servo experimental system was mounted to a two-axis swing platform which was used to simulate the attitude change of the aircraft. The composition of the experimental system is shown in Figure 6, and photograph of the experimental platform is shown in Figure 7. It is composed of a servo motor, a three-axis gyroscope, an incremental encoder, an absolute encoder, visible light camera, image tracker, position control and drive circuit, and so on. The high-precision three-axis gyroscope is employed for measurement of the angular velocity of driven pitching frame. In order to compare the angle transmission error between the driving and driven shafting due to the backlash, driving encoder and driven encoder are used to measure the angle value of the motor and the load. Due to the limitation of the installation of the experimental structure, driving encoder is a relative incremental encoder, and driven encoder is an absolute encoder. The parameters of the encoders are shown in Table 1. Angle velocity is collected through the RS422 serial port of the DSP (TMS320F28335), and angle is realized using the SPI interface and EQEP module. The sampling frequency for the angle is $10 \mathrm{kHz}$, whereas the sampling frequency for the gyroscope is $2 \mathrm{kHz}$ with a baud rate of $921.6 \mathrm{kbps}$. The reduction ratio of servo motor is $141: 1$, and reduction ratio of shafting is $3: 1$. DSP is mainly used to implement the control strategy and communicate with the image tracker to get the image miss distance, which is used to observe the effect of backlash compensation. The image is displayed and observed on PC by the image decider. All programs are programmed in the $\mathrm{C}$ language.

\section{Experimental Results and Discussion}

In order to verify the effectiveness and superiority of the extend disturbance observer (EDOB)-based robust slidingmode control, experiments compared the step data and the sinusoidal data of velocity under the same backlash, in which the velocity is $10^{\circ} / \mathrm{s}$ (unit: degrees per second) and sinusoidal input $v=3 \sin (2 \pi \times 0.2 t), \quad v=5 \sin (2 \pi \times 0.5 t), \quad$ and $v=3 \sin (2 \pi \times t)$ considering the actual aviation application conditions. As the same condition, we get the data of velocity and position under different strength sinusoidal disturbance conditions added by the swing platform, and the different strength and frequency include $d=3 \sin (2 \pi \times 0.2 t)$ and $d=$ $6 \sin (2 \pi \times 0.2 t)$ (unit: degrees). The following three methods are compared in the experiment: the traditional PI method, the traditional nonlinear extended disturbance observer (NEDOB), and the EDOB-based SMC. According to practical engineering experience, the parameters of the traditional PI method are chosen as $k_{p}=200$ and $k_{i}=1$ [21]. The main parameters of the position servo system model are as follows: $J_{e}=0.0004 \mathrm{~kg} / \mathrm{m}^{2}, \quad J_{m}=0.0058 \mathrm{~kg} / \mathrm{m}^{2}$, $\widehat{B}_{e}=0.003 \mathrm{~N} \cdot \mathrm{m} \cdot \mathrm{s} / \mathrm{rad}, \widehat{B}_{m}=0.01 \mathrm{~N} \cdot \mathrm{m} \cdot \mathrm{s} / \mathrm{rad}, i=3: 1$, and $K=0.2 \mathrm{~N} \cdot \mathrm{m} / \mathrm{rad}$.

As we know $x=\dot{\theta}_{m}$, the dynamic model of the backlash position servo system can be expressed as follows:

$$
\dot{x}=-1.724 x-2.069 \ddot{\theta}_{e}+1.552 \dot{\theta}_{e}+172.41 U+\tilde{d},
$$

where $\tilde{d}=172.41\left(3 T_{d 1}+3 d+T_{d 2}\right)$

Setting in the EDOB-based SMC, the order of the observer is the second order and then the observer is

$$
\left\{\begin{array}{l}
\dot{\omega}=-1.724 x-2.069 \ddot{\theta}_{e}+1.552 \dot{\theta}_{e}+172.41 U+\hat{\widetilde{d}} \\
\hat{\widetilde{d}}=\omega_{1}+m_{1} x, \\
\dot{\omega}_{1}=-m_{1} \dot{\omega}+v_{1}+\dot{\overrightarrow{\tilde{d}}} \\
\dot{\widetilde{\tilde{d}}}=\omega_{2}+m_{2} x \\
\dot{\omega}_{2}=-m_{2} \dot{\omega}+v_{2},
\end{array}\right.
$$

and the parameter takes $m_{1}=m_{2}=100, k=0.2, h=1$, and $\tau=0.5$. The parameters of the traditional nonlinear extended disturbance observer (NEDOB) [22] are $l_{1}=l_{2}=100$.

The experimental results of the PI, NEDOB, and the EDOB-based SMC under the velocity $10 \%$ step input are shown in Figures 8-10. Figures 8(a), 9(a), and 10(a) show the speed signal of the gyro output, and Figures 8(b), 9(b), and 10(b) show the position of the driven gear obtained by the driven encoder in the backlash stage. The RMS value of the position formula is as follows:

$$
\mathrm{RMS}=\left[\frac{1}{n-1} \sum_{i=1}^{n}\left(x_{i}-\bar{x}\right)^{2}\right]^{(1 / 2)} .
$$

The experimental results show that the response curve of this method is smoother in the steady-state phase of speed step, the maximum steady-state error of PI control is $1.58^{\circ} / \mathrm{s}$, and NEDOB is $0.98^{\circ} / \mathrm{s}$, and compared with them, EDOBbased SMC is $0.37^{\circ} / \mathrm{s}$. In the process of step dynamic response, the control overshoot of PI control, NEDOB, and EDOB-based SMC is $31.8 \%, 15.1 \%$, and $5.2 \%$, and it can be proved that the dynamic overshoot of this method is the minimum, which can realize the smooth movement speed and reduce the oscillation when backlash exists. Through the data comparison, it can be seen that in the dynamic and steady process of servo step, EDOB-based SMC effectively suppresses the oscillation impact caused by the backlash and makes the gear transmission more stable. In order to better verify the backlash transmission compensation effect, under 


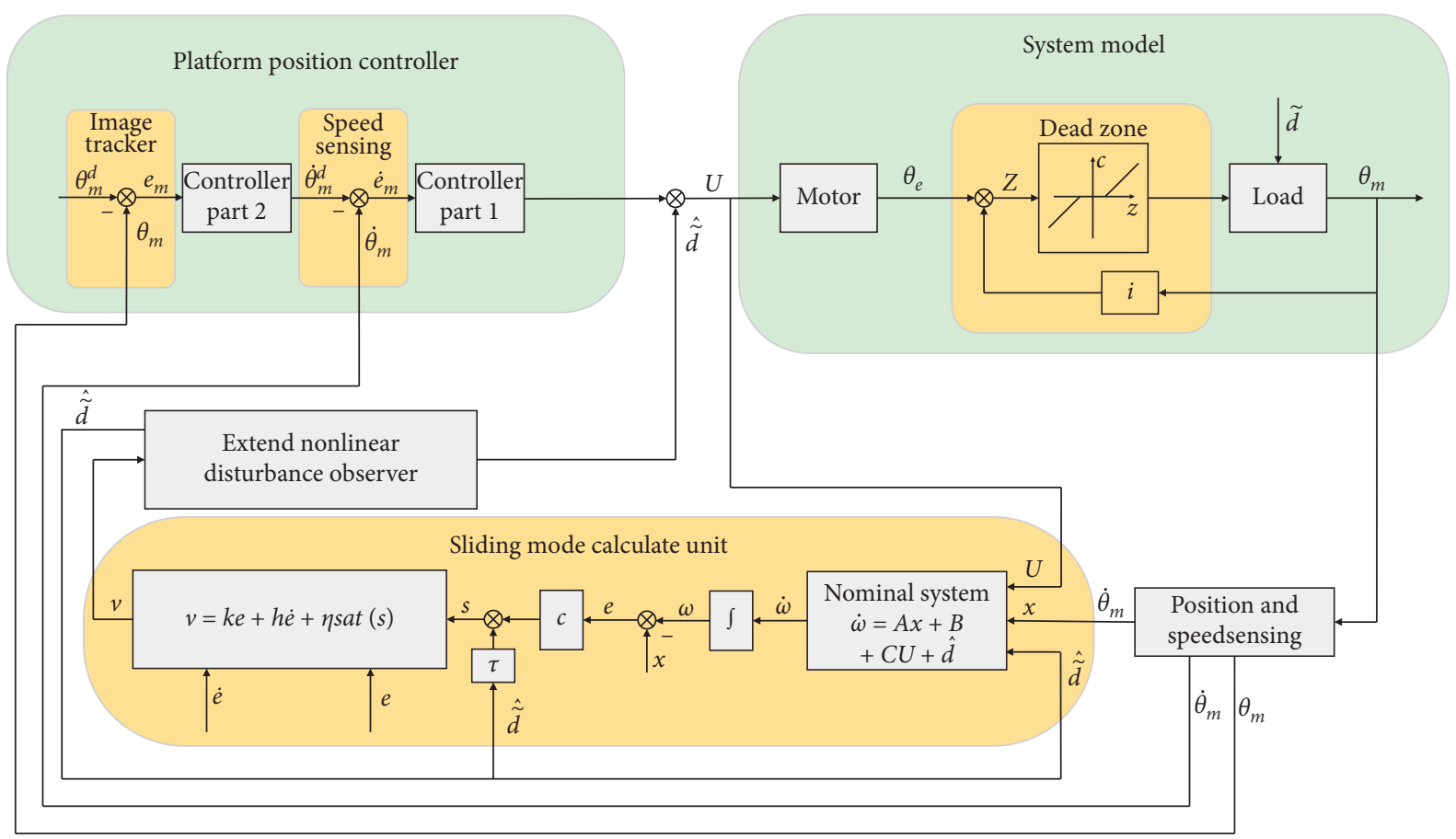

FIGURE 5: The extend nonlinear disturbance observer based on the sliding mode.

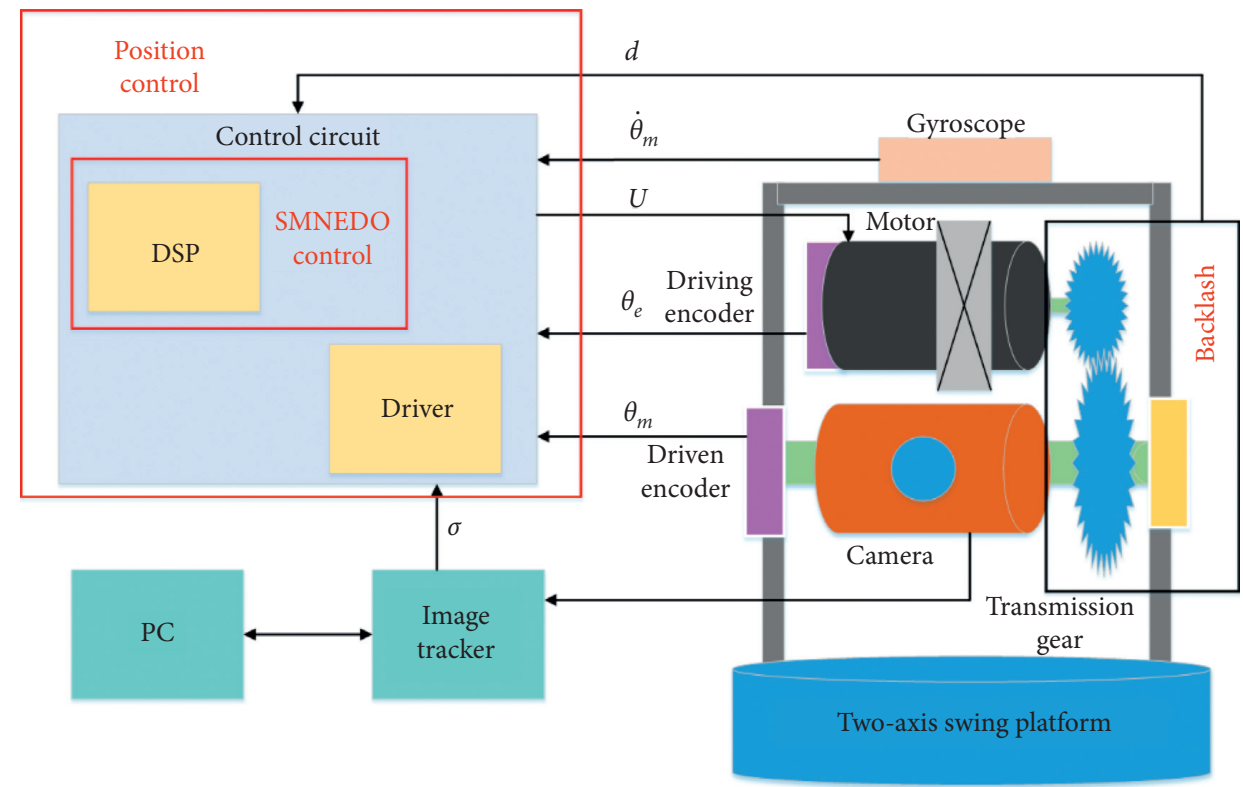

Figure 6: Configuration of the DSP-based experimental setup.

the velocity $v=3 \sin (2 \pi \times 0.2 t), v=5 \sin (2 \pi \times 0.5 t)$, and $v=3 \sin (2 \pi \times t)$ input, the experimental data are shown in Figures 11-13.

The experimental results demonstrate that, at the speed $v=3 \sin (2 \pi \times 0.2 t), \quad v=5 \sin (2 \pi \times 0.5 t), \quad$ and $v=3 \sin (2 \pi \times t)$, EDOB-based SMC achieves a satisfactory speed response performance. At the speed positive and negative switching time, EDOB-based SMC makes the speed transition smoother and shortens the response time. Compared with the PI method $(0.01 \mathrm{~s} v=3 \sin (2 \pi \times 0.2 t))$ $(0.024 \mathrm{~s} v=5 \sin (2 \pi \times 0.5 t))(0.055 \mathrm{~s} v=3 \sin (2 \pi \times t))$ and the NEOD method $(0.008 \mathrm{~s} v=3 \sin (2 \pi \times 0.2 t))(0.011 \mathrm{~s}$ $v=5 \sin (2 \pi \times 0.5 t))(0.035 \mathrm{~s} v=3 \sin (2 \pi \times t))$, the speed response time of the EDOB-based SMC is $0.006 \mathrm{~s}(v=3 \sin (2 \pi \times 0.2 t)), 0.008 \mathrm{~s}(v=5 \sin (2 \pi \times 0.5 t))$, and $0.025 \mathrm{~s}(v=3 \sin (2 \pi \times t))$, which reduces by $40 \%$ and 


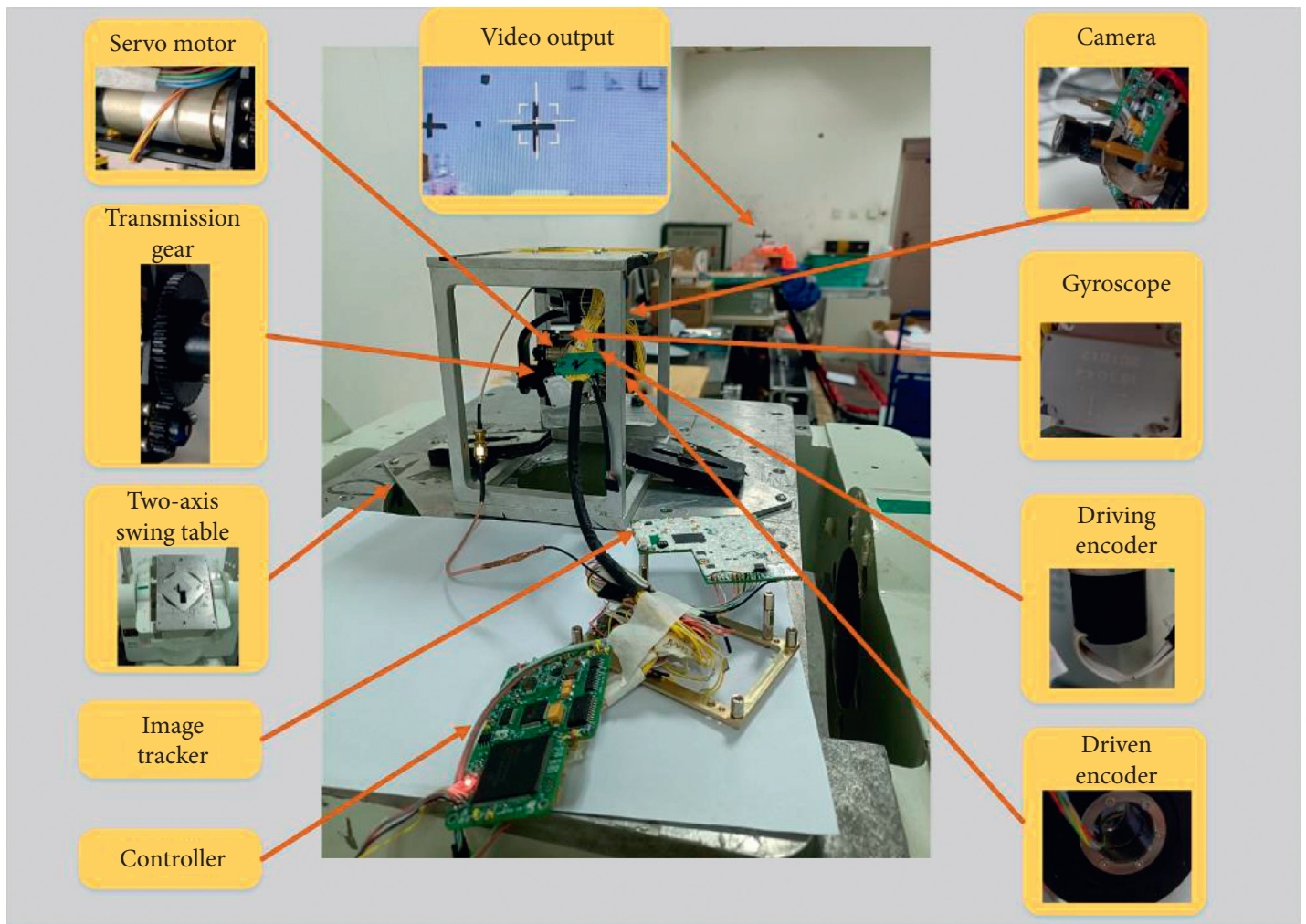

Figure 7: Photograph of the experimental platform.

Table 1: Parameters of driving encoder and driven encoder.

\begin{tabular}{lcc}
\hline Description & & Value \\
\hline Resolution ratio & Driving encoder & Driven encoder \\
Frequency range & $2^{21}$ & $2^{19}$ \\
Data output mode & $875 \mathrm{kHz}$ & $1 \mathrm{MHz}$ \\
Supply voltage & EQEP & $\mathrm{SSI}$ \\
Maximum operational speed & $4.5 \sim 5.5 \mathrm{~V}$ & $4.75 \sim 5.25 \mathrm{~V}$ \\
Operating temperature range & $2000 \mathrm{rpm}$ & $3500 \mathrm{rpm}$ \\
\hline
\end{tabular}

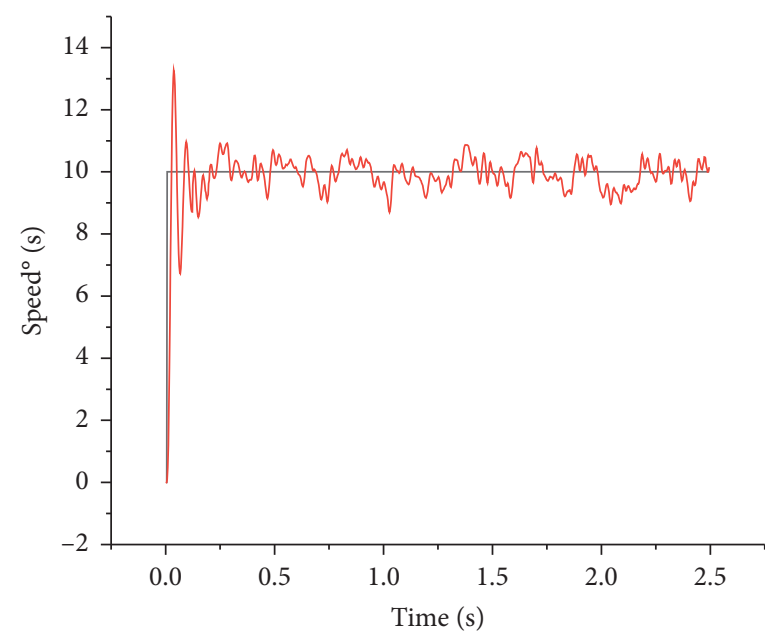

- Speed step input Speed of gyro

(a)

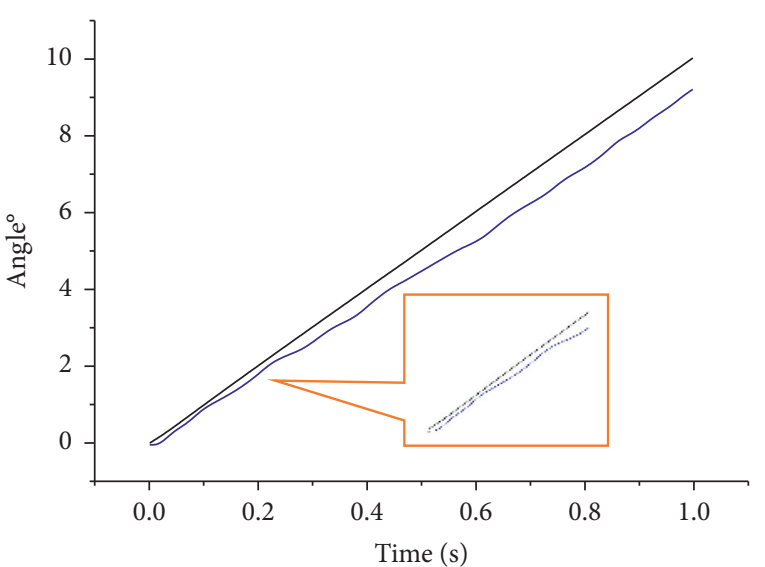

— Driving encoder data
D Driven encoder data

(b)

FIGURE 8: Experiment results of PI control under the step 10\%: (a) speed of the gyro; (b) the angle of the driving gear and the driven gear. 


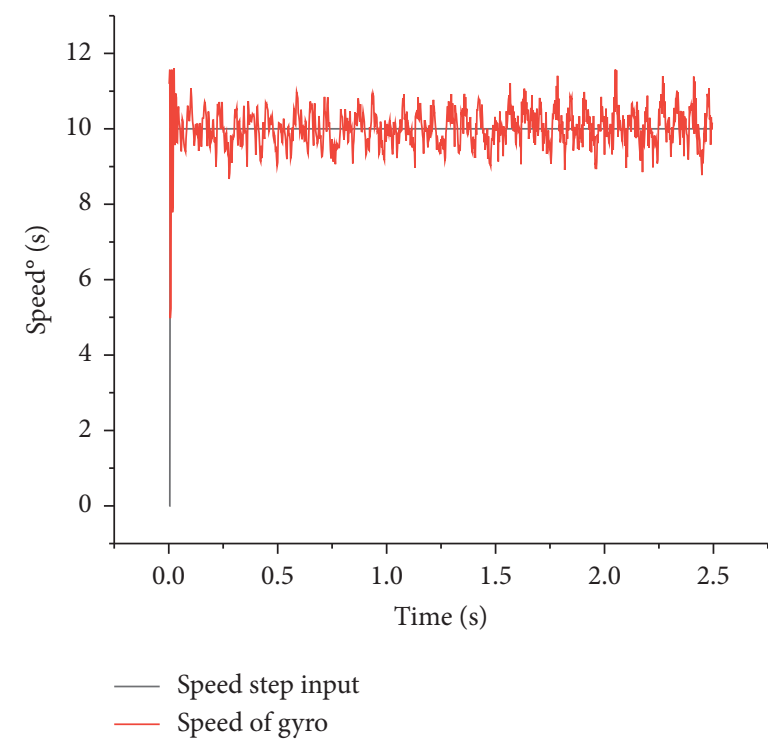

(a)

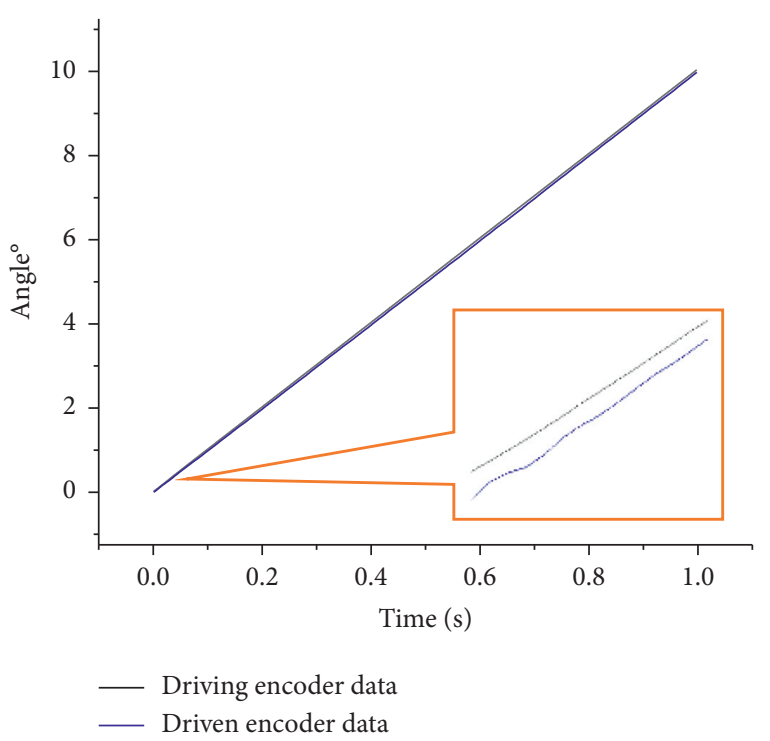

(b)

Figure 9: Experiment results of NEDOB under the step 10\%s: (a) speed of the gyro; (b) the angle of the driving gear and the driven gear.

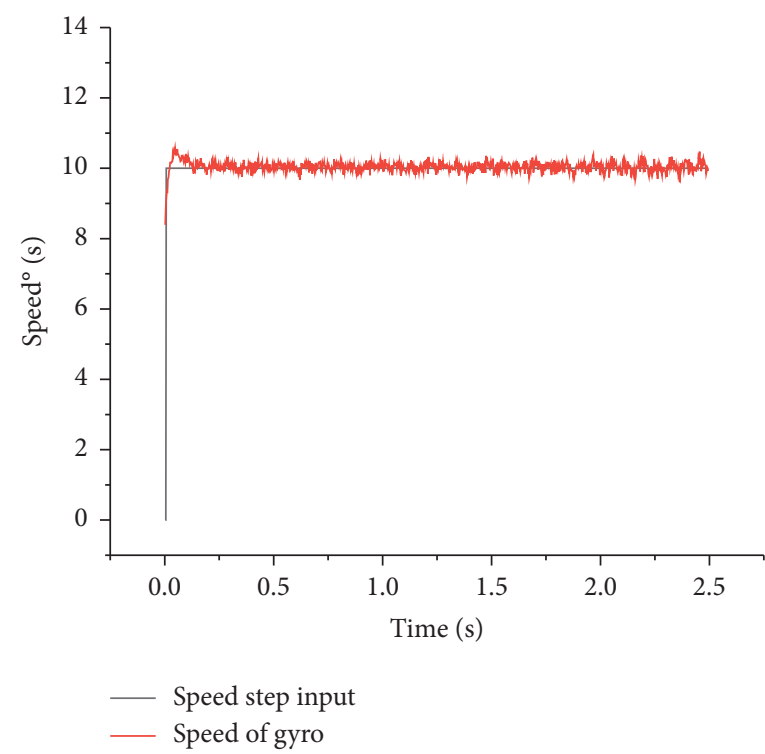

(a)

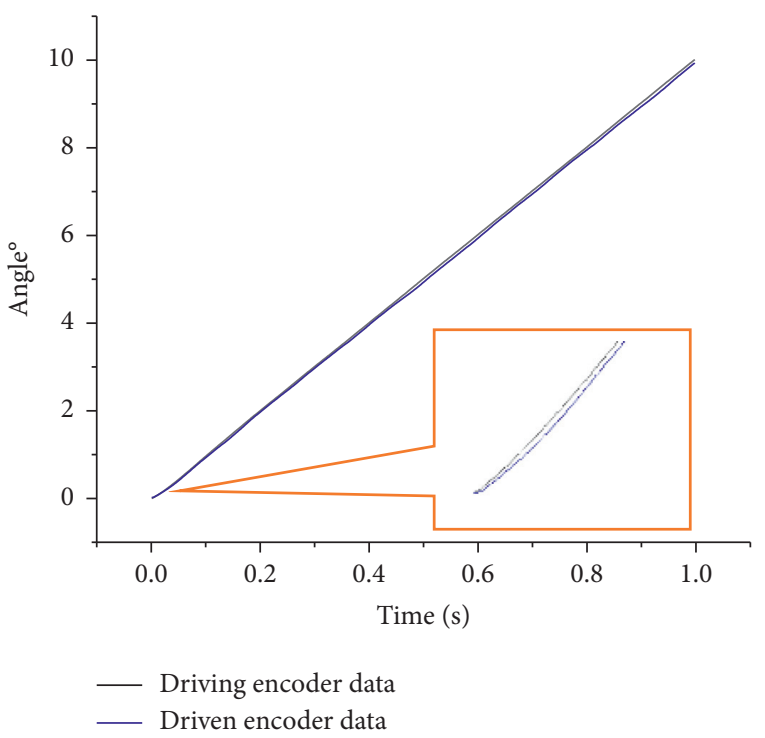

(b)

Figure 10: Experiment results of EDOB-based SMC under the step $10 \%$ s: (a) speed of the gyro; (b) the angle of the driving gear and the driven gear.

$25 \%$; $66.6 \%$ and $27.2 \%$; and $54.5 \%$ and $28.5 \%$ within $\pm 0.05 \%$. At the same time, the number of motion velocity oscillations is significantly reduced.

Figures 14-19 show the experiment results in the different frequencies and amplitude disturbance signal $d=3 \sin (2 \pi \times 0.2 t)$ and $d=6 \sin (2 \pi \times 0.2 t)$, which is with the maximum acceleration of $4.73^{\circ} / \mathrm{s}^{2}$ and $9.46^{\circ} / \mathrm{s}^{2}$. There are three reasons for choosing sinusoidal form as disturbance. Firstly, sinusoidal function as disturbance input is intuitive and controllable; secondly, it is easy to realize on the twoaxis swing platform; thirdly, in actual aviation flight, the actual shaking form of aircraft is similar to sinusoidal motion.

From the experimental results presented, it is evident that EDOB-based SMC has obvious advantages, which effectively compensates the negative influence of the backlash in the process of suppressing the external disturbance. The RMS value of the stable angle error under different disturbance conditions is shown in Table 2. It can be seen from the data in Table 2, the speed changes direction and the driving gear carries out noncontact acceleration movement between the backlash, resulting in greater speed impact, 


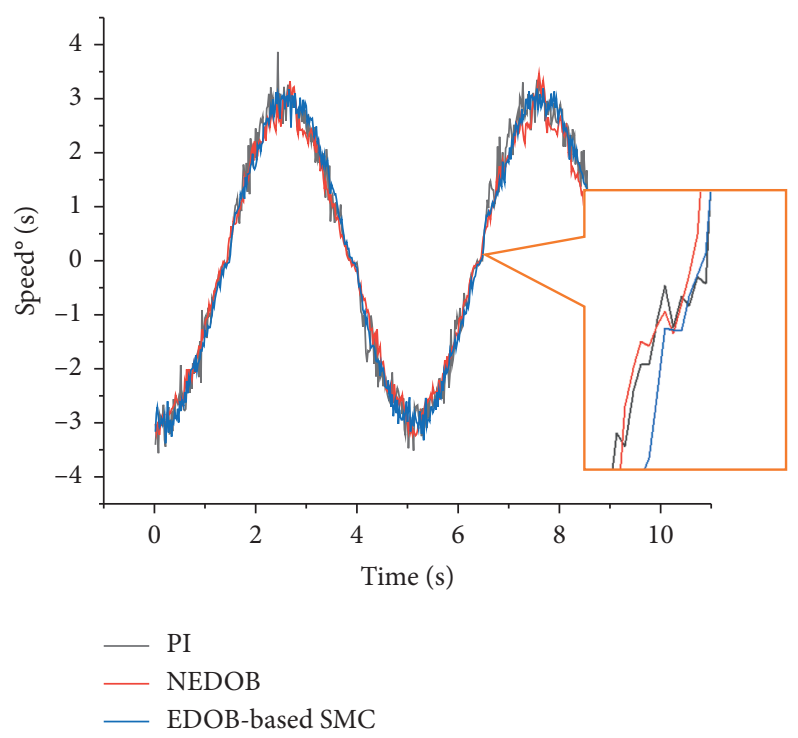

FIgURE 11: Speed comparison of PI, NEDOB, and EDOB-based SMC under the $v=3 \sin (2 \pi \times 0.2 t)$.

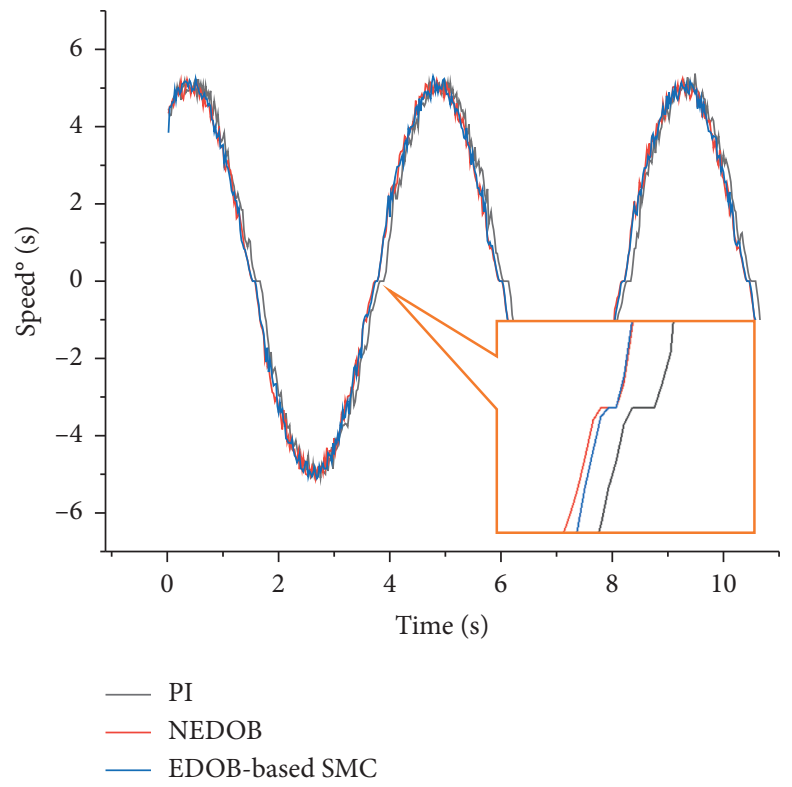

FIgURE 12: Speed comparison of PI, NEDOB, and EDOB-based SMC under the $v=5 \sin (2 \pi \times 0.5 t)$. 


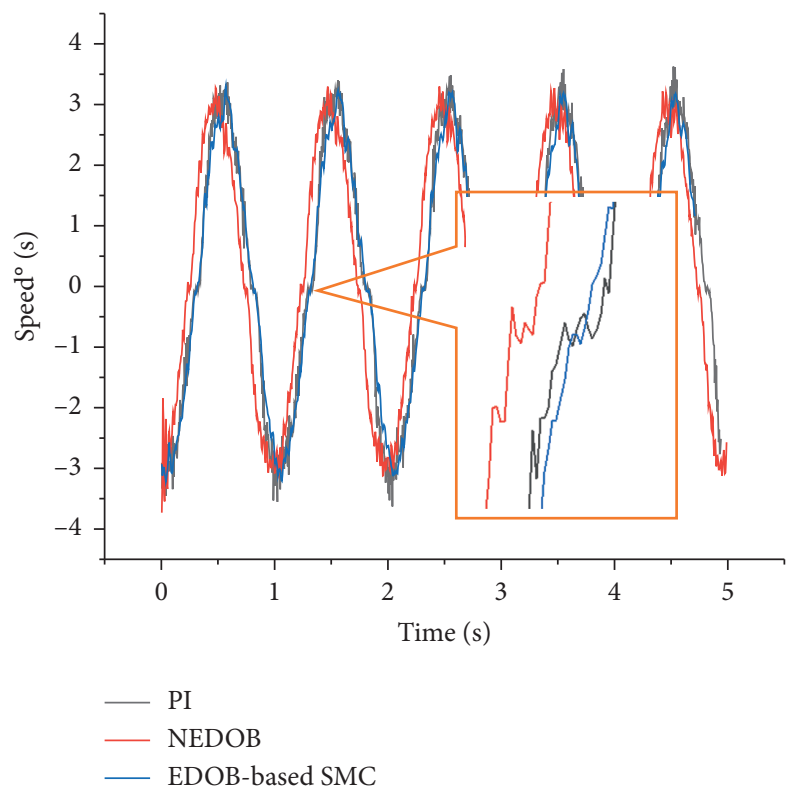

FIgURE 13: Speed comparison of PI, NEDOB, and EDOB-based SMC under the $v=3 \sin (2 \pi \times t)$.

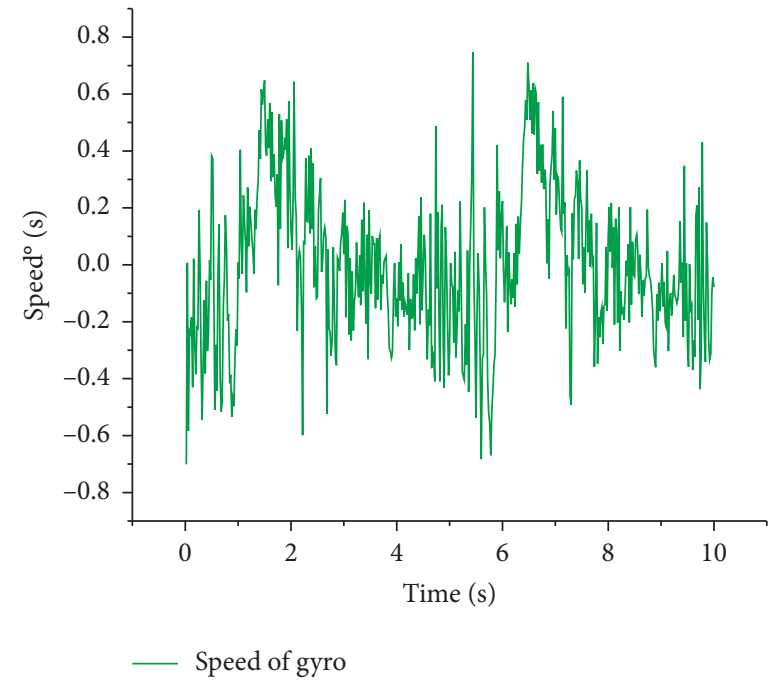

(a)

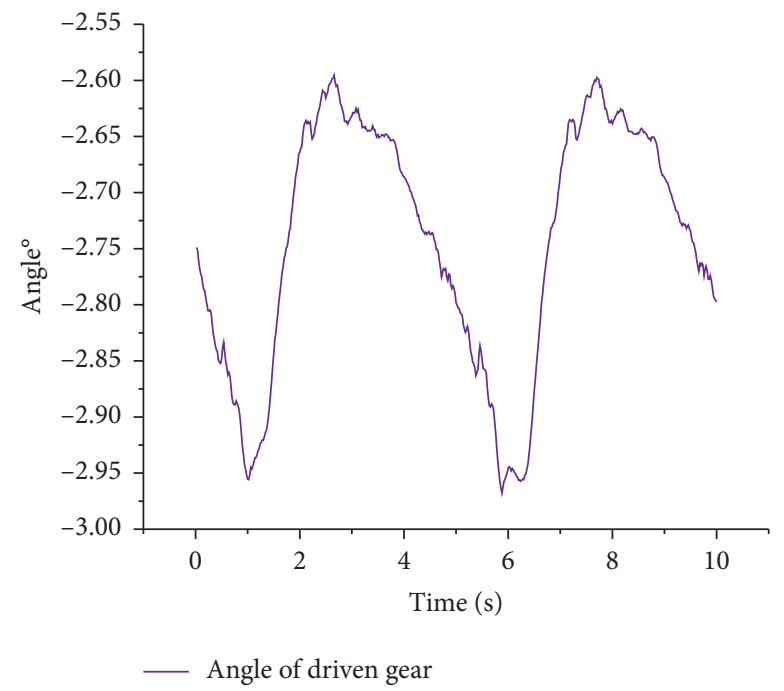

(b)

FIgURe 14: Experiment results of PI control under $d=3 \sin (2 \pi \times 0.2 t)$ : (a) speed of the gyro; (b) stable angle error of the driven gear. 


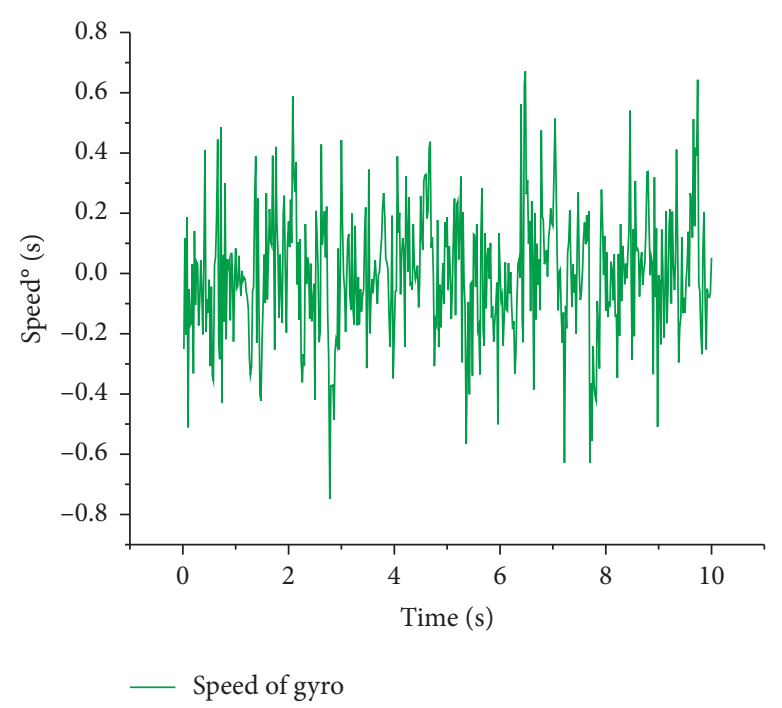

(a)

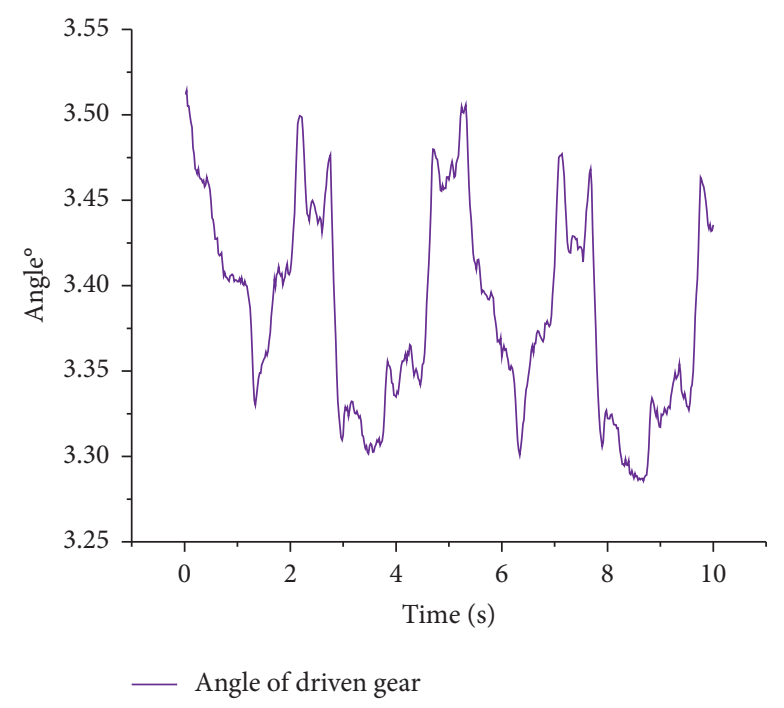

(b)

FIgURE 15: Experiment results of NEDOB under $d=3 \sin (2 \pi \times 0.2 t)$ : (a) speed of the gyro; (b) stable angle error of the driven gear.

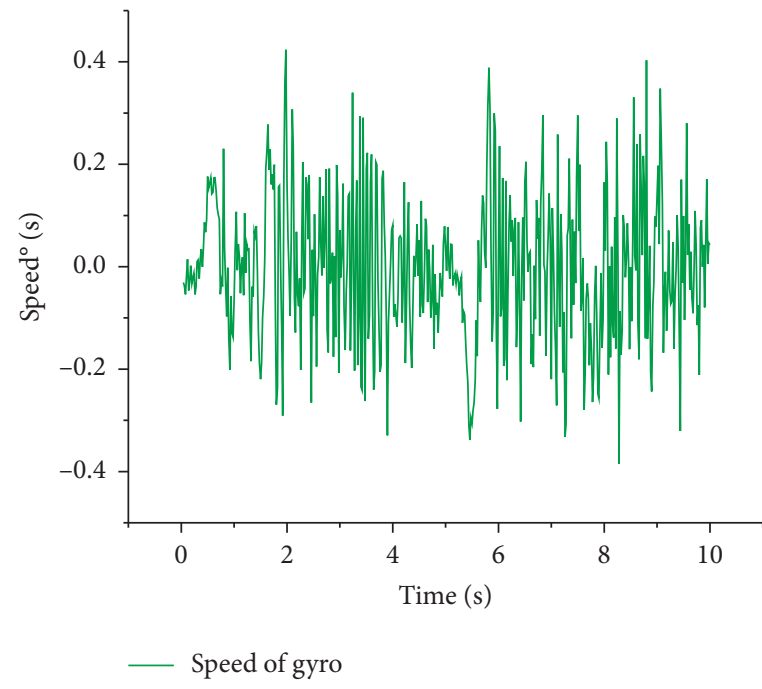

(a)

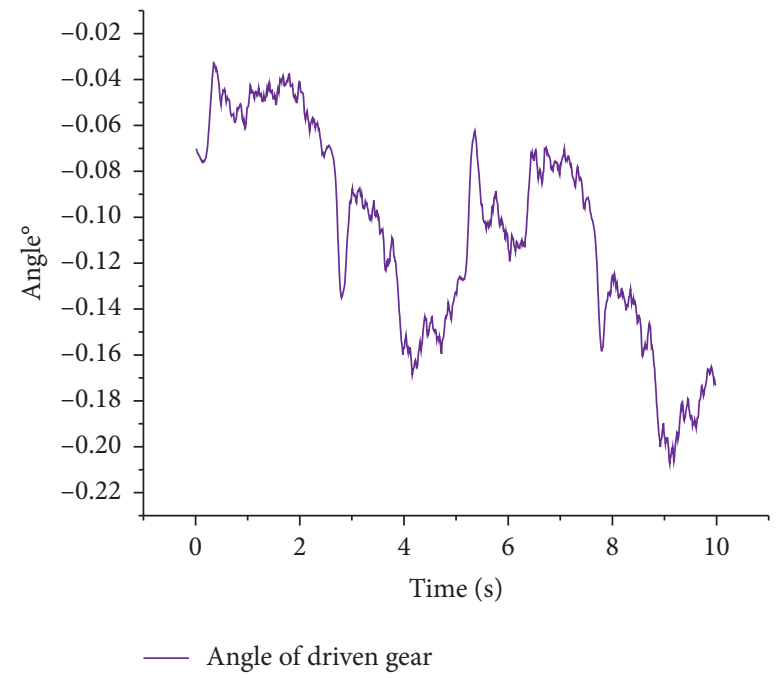

(b)

FiguRE 16: Experiment results of EDOB-based SMC under $d=3 \sin (2 \pi \times 0.2 t)$ : (a) speed of the gyro; (b) stable angle error of the driven gear. 


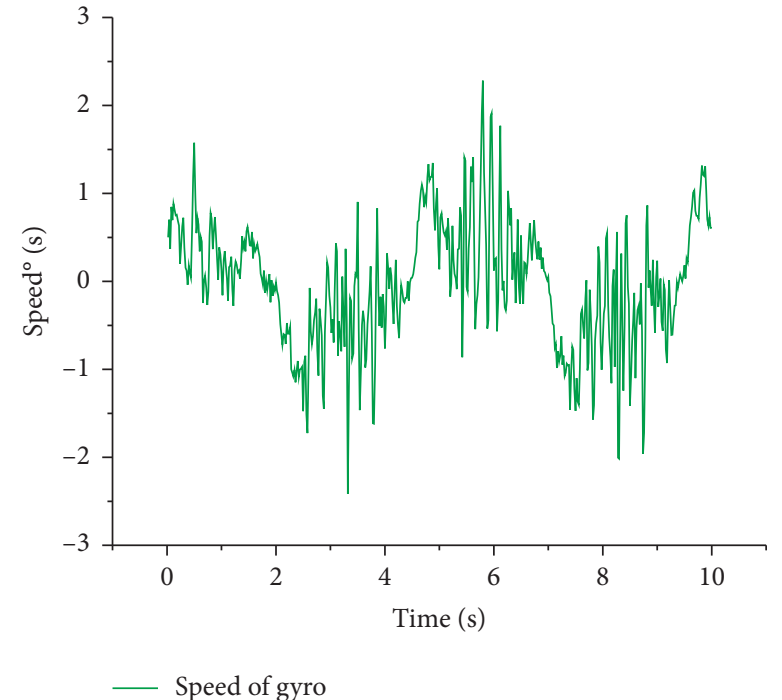

(a)

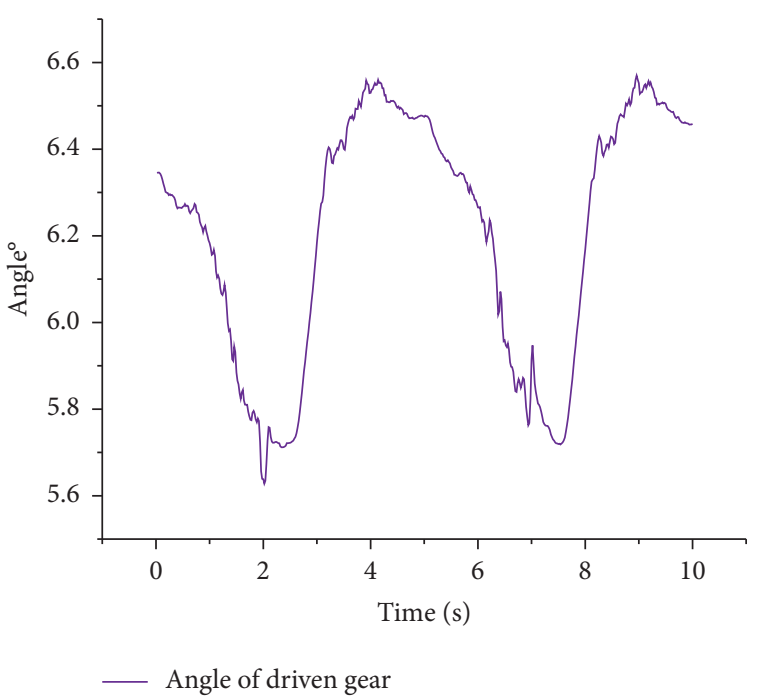

(b)

Figure 17: Experiment results of PI control under $d=6 \sin (2 \pi \times 0.2 t)$ : (a) Speed of the gyro; (b) stable angle error of the driven gear.

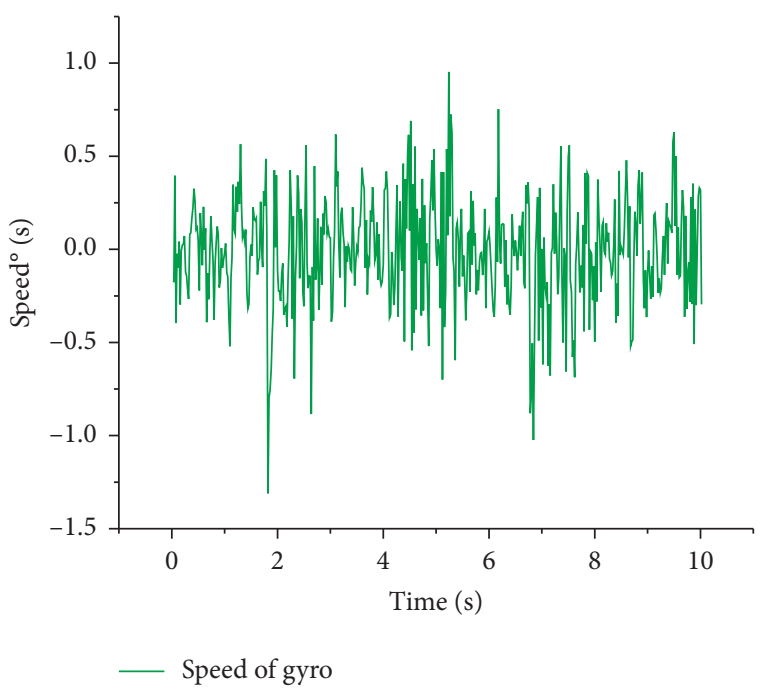

(a)

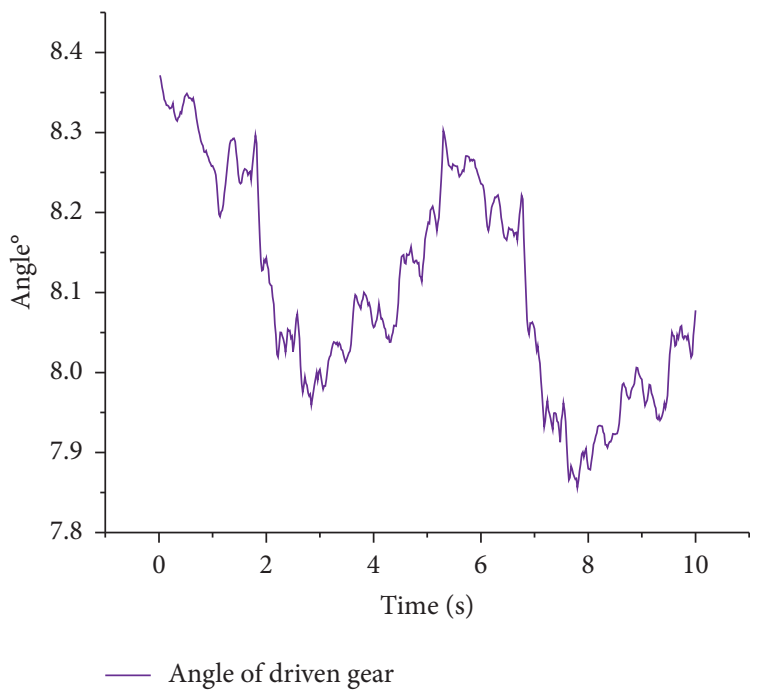

(b)

FIGURE 18: Experiment results of NEDOB under $d=6 \sin (2 \pi \times 0.2 t)$ : (a) speed of the gyro; (b) stable angle error of the driven gear. 


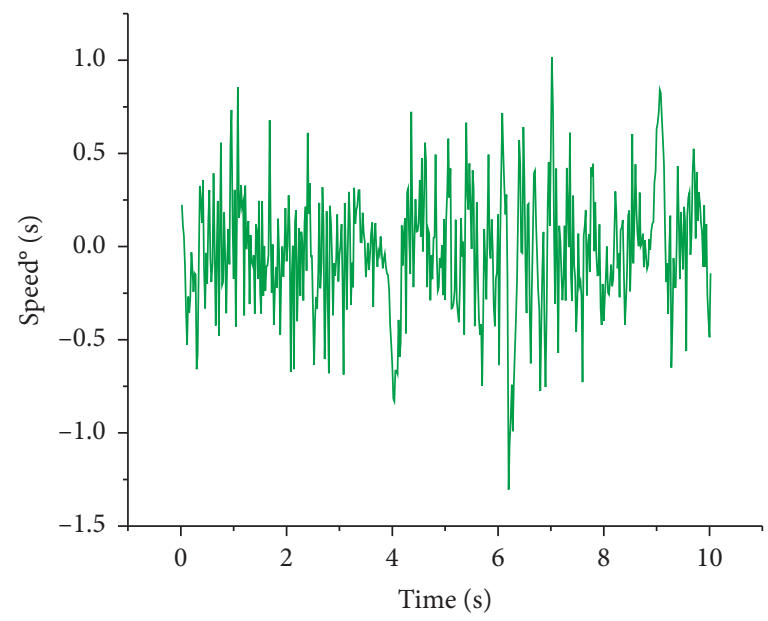

— Speed of gyro

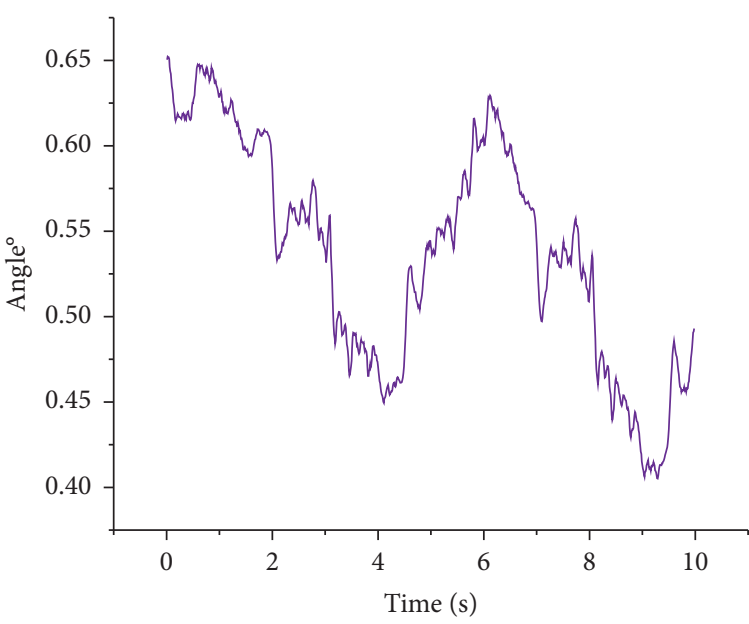

Angle of driven gear

(b)

Figure 19: Experiment results of EDOB-based SMC under $d=6 \sin (2 \pi \times 0.2 t)$ : (a) speed of the gyro; (b) stable angle error of the driven gear.

TABLE 2: Comparison results of the stable angle error.

\begin{tabular}{lcc}
\hline \multirow{2}{*}{ Description } & \multicolumn{2}{c}{ Different disturbance conditions } \\
& $d=3 \sin (2 \pi \times 0.2 t)$ & $d=6 \sin (2 \pi \times 0.2 t)$ \\
\hline PI control & 66 urad & 157 urad \\
NEDOB & 39.9 urad & 89.8 urad \\
EDOB-based SMC & 25.3 urad & 36.3 urad \\
\hline
\end{tabular}

which seriously affects the interference isolation performance of the stable platform. The EDOB-based SMC method reduces the backlash speed impact and ensures that the platform has higher LOS stability.

\section{Conclusions}

An extend disturbance observer (EDOB)-based robust sliding-mode control which is used to compensate backlash dead zone nonlinearity is presented in this paper, for the purpose of compensating backlash characteristics which causes instability, hysteresis, even commutation jump, and impact oscillation of the platform, so it can isolate the influence of various external interference and aircraft attitude motion on the optical load LOS. EDOB-based SMC combines extend disturbance observer (EDOB) and robust sliding-mode control to compensate backlash speed impact and enhances the robustness of modified disturbance observer on the backlash characteristics, to achieve better inertial stability. Experimental investigations were conducted on a DSP-based platform with backlash in the pitch shafting. In order to better verify the backlash transmission compensation effect, the servo system takes shafting motion under the step and sinusoidal input and in the different frequency and amplitude disturbance signal to test the anti-interference ability of this method. Comparing the PI method and NEDOB, the effectiveness of the proposed EDOB-based SMC scheme was demonstrated by the experimental results.

\section{Data Availability}

The datasets used and analysed during the current study are available from the corresponding author upon reasonable request.

\section{Conflicts of Interest}

The authors declare that there are no conflicts of interest.

\section{Acknowledgments}

This study was funded by the general program of National Natural Science Foundation of China, No. 61675202.

\section{References}

[1] L. Q. Wu, "The elimination ways of the gear-driven clearance," Machine Tool \& Hydraulics, vol. 5, pp. 187-188, 2005.

[2] H. A. Hu, "A nonlinear compensation method for backlash transmission backlash in servo system," IEEE Changchun Inst of Optics Fine Mechanics \& Physics, vol. 2, pp. 5505-5507, 2011.

[3] G. Tao and P. V. Kokotovic, "Adaptive control of system with unknown output backlash," IEEE Transactions on Automatic Control, vol. 40, no. 2, pp. 326-330, 1995.

[4] A. Azenha and M. Tenreiro, "Variable structure control of system with nonlinear friction and dynamic backlash," IFAC Proceedings Volumes, vol. 1, pp. 237-242, 1996.

[5] L. W. Li, X. M. Ren, M. L. Wang et al., "Identification and control of sandwich system with backlash," in Proceedings of the 36th Chinese Control Conference, pp. 26-28, Dalian, China, July 2017.

[6] M. Nordin, P. O. Gutman, and R. Selmic, "Non-linear speed control of elastic system with backlash," in Proceedings of the 39th IEEE Conference on Decision and Control, pp. 4060-4065, Sydney, Australia, December 2000.

[7] N. J. Ahmad and F. Khorrami, "Adaptive control of system with backlash hysteresis at the input," in Proceedings of the 
American Control Conference, pp. 3018-3022, San Diego, CA, USA, June 1999.

[8] X. L. Zhang, C. L. Fu, and L. J. Jia, "Fuzzy PID inverse compensation for mechanic system with input backlash," in Proceedings of the Chinese Automation Congress (CAC), pp. 4036-4040, Hangzhou, China, November 2019.

[9] J. Garnica, J. Marrugo, and C. E. Horacio, "ADRC inverse compensation for mechatronic system with output backlash \& Single encoder," in Proceedings of the 4th International Congress of Mechatronics Engineering and Automation, Cartagena, Colombia, November 2020.

[10] K. F. Zhang, X. L. Xu, F. Wu et al., "Robust adaptive backstepping control for missile servo control system with backlash and disturbances," in Proceedings of the 39th Chinese Control Conference, pp. 617-622, Shenyang, China, July 2020.

[11] G. Lai, Z. Liu, Y. Zhang, C. L. P. Chen, and S. Xie, "Asymmetric actuator backlash compensation in quantized adaptive control of uncertain networked nonlinear systems," IEEE Transactions on Neural Networks and Learning Systems, vol. 28, no. 2, pp. 294-307, 2017.

[12] R. Merzouki, J. C. Cadiou, and K. M'Sirdi, "Compensation of backlash effects in an electrical actuator," in Proceedings of the 2003 European Control Conference, pp. 2511-2516, Cambridge, UK, September 2003.

[13] I. Kolnik and G. Agranovich, "Backlash compensation for motion system with elastic transmission," in Proceedings of the 2012 IEEE 27th Convention of Electrical and Electronics Engineers, pp. 1-5, Eilat, Israel, November 2012.

[14] K. Wu and X. M. Ren, "Fast recursive dynamic sliding control based on improved extended state observer for Dual-motor servo system with backlash nonlinearity," in Proceedings of the 2014 Sixth International Conference in Intelligent Human System and Cybernetics, pp. 173-176, Zhejiang, China, August 2014.

[15] W. H. Chen, D. Liu, Y. Zhang et al., "Fast recursive dynamic sliding control based on improved extended state observer for Dual-motor servo system with backlash nonlinearity," in Proceedings of the 2014 Sixth International Conference in Intelligent Human System and Cybernetics, pp. 173-176, Zhejiang, China, August 2014.

[16] J. Yang, W.-H. Chen, and S. Li, "Non-linear disturbance observer-based robust control for systems with mismatched disturbances/uncertainties," IET Control Theory \& Applications, vol. 5, no. 18, pp. 2053-2062, 2011.

[17] D. Ginoya, P. D. Shendge, and S. B. Phadke, "Sliding mode control for mismatched uncertain systems using an extended disturbance observer," IEEE Transactions on Industrial Electronics, vol. 61, no. 4, pp. 1983-1992, 2014.

[18] J. Yang, S. Li, and X. Yu, "Sliding-mode control for systems with mismatched uncertainties via a disturbance observer," IEEE Transactions on Industrial Electronics, vol. 60, no. 1, pp. 160-169, 2013.

[19] T. C. Kuo, S. H. Chang, and Y. J. Huang, "Sliding mode control governed by adaptive tuning law for uncertain nonlinear system," in Proceedings of the 2006 IEEE International Conference on Mechatronics and Automation, pp. 1049-1053, Henan, China, June 2006.

[20] C. L. Hwee, D. Oetomo, G. Alici et al., "Special class of positive definite functions for formulating adaptive micro/nano manipulator control," in Proceedings of the IEEE International Workshop on Advanced Motion Control, pp. 517-522, Yokohama, Japan, August 2006.

[21] Z. Zhou, B. Zhang, and D. Mao, "Robust sliding mode control of PMSM based on rapid nonlinear tracking differentiator and disturbance observer," Sensors, vol. 18, no. 4, pp. 1031-1050, 2018.

[22] L. N. Tan, G. D Jin, C. Liu et al., "Extend disturbance observer for nonlinear system based on sliding-mode theory," in Proceedings of the 2017 IEEE 2th Information Technology, Networking, Electronic and Automation Control Conference, pp. 828-832, Chongqing, China, March 2017.

[23] R. Merzouki and J. C. Cadiou, "Estimation of backlash phenomenon in the electromechanical actuator," Control Engineering Practice, vol. 13, no. 8, pp. 973-983, 2005.

[24] Y. Shui and J. L. Wei, "The influence and compensation of gear backlash on electric load system," in Proceedings of the 2017 2nd International Conference on Frontiers of Sensors Technologies, pp. 479-483, Shenzhen, China, April 2017.

[25] Y. Q. Xiao and L. M. Chen, "Adaptive fuzzy sliding mode control based on backstepping method for position servo system with backlash," Machine Building \& Automation, vol. 6, pp. 187-191, 2016.

[26] Y. Han, P. Li, and Z. Q. Zheng, "Sliding-mode and Backstepping control for output tracking systems with unmatched uncertainties via a disturbance observer," in Proceedings of the 2016 14th International Workshop on Variable Structure Systems (VSS), pp. 308-311, Nanjing, China, June 2016.

[27] J. Zhou and J. L. Zhao, "Based on the extended disturbance observer of reentry vehicle terminal sliding mode control," Journal of Northwestern Polytechnical University, vol. 10, pp. 818-822, 2016. 